DOI: $10.1002 /$ adom.201200011

\title{
Magnetoplasmonics: Combining Magnetic and Plasmonic Functionalities
}

By Gaspar Armelles, Alfonso Cebollada, Antonio García-Martín, and María Ujué González

Prof. G. Armelles, Prof. A. Cebollada, Dr. A. García-Martín, Dr. M. U. González

IMM-Instituto de Microelectrónica de Madrid (CNM-CSIC)

Isaac Newton 8, PTM, E-28760 Tres Cantos, Madrid, Spain

E-mail: alfonso@imm.cnm.csic.es

Keywords: ((Five maximum: Magnetoplasmonics, magneto-optics, plasmonics, active plasmonics, non-reciprocity))

\section{$\underline{\text { Abstract }}$}

Nanosystems with combined magnetic and plasmonic functionalities have in recent years become an active topic of research. By an adequate internal architecture of the constituting components, the magneto-optical activity of these systems can be largely increased due to the electromagnetic field enhancement associated with the plasmon resonance. Simultaneously, the magnetic functionality permits the control of the plasmonic properties by an external magnetic field, which allows the development of active plasmonic devices. These materials find applications for example in gas and biosensing areas and in integrated photonic devices for telecommunications. In the present work we review the state of the art and current understanding of the phenomenology associated with magnetoplasmonic structures where magnetism and plasmonics are intertwined.

\section{Introduction}

The term magnetoplasmon, or magnetoplasma surface wave, was first introduced in the early ' 70 s, motivated by the interest on surface polaritonic waves that the relatively recent discovery of surface plasmons in metals and degenerate semiconductors attracted. ${ }^{[1,2,3,4,5,6]}$ At that time, the effect of an external magnetic field on the dielectric 
function of the electron plasma seeded a number of fundamental investigations focused on the magnetic field modification of the dispersion relation of surface plasma and coupled surface plasma-phonon waves. ${ }^{[1,2,3]}$ Later in the ' $80 \mathrm{~s}{ }^{[7,8,9,10,11,12,13,14,15,16]}$ and '90s [17,18,19,20,21], plasmon resonances and magnetism found another important interaction niche in the, by then, growing and strategic field of magnetic recording, and in particular of magneto-optical (MO) recording. Different groups studied the possible enhancement effects that bulk and surface plasmons could produce on the MO activity of a number of materials systems.

The arrival of the new century brought the generalization of nanofabrication tools and nanotechnology, providing new nanostructures where again the interaction between magnetic field, MO properties and surface plasmons could be revisited, mainly in the visible and near-infrared range of the electromagnetic spectrum. Moreover, the theoretical tools devoted to the analysis of the optical response of nanostructures in the visible range have been recently adapted to deal with the MO effects. ${ }^{[22,23,24,25,26,27,28,29,30]}$ As a consequence, this topic has become an active area of investigation and an increasing number of research groups are exploring this phenomenology from the experimental and theoretical viewpoints. This renewed interest is strongly linked to the development and fast growth of the so-called plasmonics, a subfield of nanooptics that aims at understanding and controlling light using the surface plasmon resonances sustained by metal nanostructures, ${ }^{[31,32]}$ as well as to the renovated interest on MO enhancement through nanostructuration ${ }^{[33,34,35]}$ and the increasing importance of magnetic nanoparticles applications and the understanding of their optical and MO properties. Indeed, and mirroring the early times, again both directions, namely the influence of a magnetic field on the surface plasmons properties and the MO enhancement associated with plasmon resonances, are the driving forces in 
the magnetoplasmonic community. The field of applications of such structures is found in areas such as sensing ${ }^{[36]}$ and telecommunications. ${ }^{[37]}$

Surface plasmons (SPs) are electromagnetic waves coupled to the collective oscillations of the surface free charges in an interface between two media with permittivities with opposite sign, typically a dielectric and a metal. ${ }^{[31]}$ Surface plasmon modes are strongly localized at the interface between these two media and can exist on a wide variety of metallic structures, such as single surfaces, thin films, nanoparticles, cylinders, etc. They can be classified into two main categories: localized surface plasmons (LSPs), ${ }^{[38,39]}$ also called particle plasmons, which are the ones sustained by entities of dimensions of the order or smaller than the wavelength of the exciting radiation; and surface plasmon polaritons (SPPs) or propagating surface plasmons, ${ }^{[40,41]}$ those supported by planar interfaces. Both kinds of plasmons are sketched in Figure 1, where the charge and electromagnetic (EM) field distribution are shown for each case, and an extinction spectrum for the LSP case and the dispersion relation curve for the SPP one are also presented. As it can be seen in Figure 1, surface plasmons are able to confine the EM field in small, nanoscopic volumes beyond the diffraction limit, which makes them suited for the development of nanophotonic devices. ${ }^{[32,42,43]}$ This strong confinement can result in huge local field enhancements which increase enormously the interaction of light with molecules or emitters located there. ${ }^{[44]}$ SPs are also highly sensitive to the optical properties (refractive index) of the dielectric media surrounding the metal, being therefore sensing one of the main and better established applications. ${ }^{[45,46]}$

A step forward in the development of plasmonics that would allow the realization of fundamental components in nanophotonic chips, such as modulators, switches or active multiplexors and couplers, is to find ways of controlling plasmon properties by using 
external agents. Important progress has been made in the last years to achieve active plasmonic configurations based on different controlling mechanisms, such as: temperature ${ }^{[47,48]}$ voltage ${ }^{[49,50,51]}$ or photons. ${ }^{[52,53,54,55]}$ This last option is, up to now, the only demonstrated one that could work at high switching speeds. A competitive alternative candidate for active plasmonics is the magnetic field, as it allows a modification of the optical properties which depends not only on its magnitude, but also on its direction. Moreover, as magnetism is intrinsically an ultrafast property, ${ }^{[56]}$ the switching speed at femtosecond levels could be attainable.

Magnetic field induced modifications of the optical properties of materials were first observed by M. Faraday $(1845)^{[57]}$ and J. Kerr $(1877,1878) .{ }^{[58,59]}$ They detected a change in the polarization state of the transmitted (Faraday effect) or reflected light (Kerr effect) when a magnetic field was applied to a glass or to a ferromagnetic material, respectively (see Figure 2). Since then, magneto-optics has played a relevant technological role in different areas, being the field of information storage an especially important one. Nowadays, these effects are used to develop optical devices such as isolators, modulators, or sensors, and many works have been focused on the development of materials with large MO activity to improve the performance of these devices and expand the applications area. Particularly in integrated optics, where the size is an important factor, the search for new materials or structures with large MO activity, low loses, and compatible with the fabrication processes is an open field of research, and plasmonic resonances could play a key role in reducing the device size, due to the enhancement of the MO response.

Let us first introduce some basic concepts on magneto-optics to present the notation and to allow the reader inexperienced in this topic following the physical mechanisms that underlie the phenomena to be presented in this review. Under the presence of a 
magnetic field the dielectric tensor of an isotropic material becomes non-diagonal, adopting the form: ${ }^{[60]}$

$$
\varepsilon=\left(\begin{array}{ccc}
\varepsilon & a \Pi_{z} & a \Pi_{y} \\
-a \Pi_{z} & \varepsilon & -a \Pi_{x} \\
-a \Pi_{y} & a \Pi_{x} & \varepsilon
\end{array}\right),
$$

where we keep the notation $\varepsilon$ for the diagonal tensor components and $a \prod_{i}$ represents the MO constant of the material $\left(\varepsilon_{m o}\right)$, being $\Pi_{i}$ the components of the applied magnetic field for paramagnetic and diamagnetic materials, and the magnetization for ferromagnetic (FM) ones (only linear terms in $\Pi$ are considered). Therefore, the effect that an external magnetic field has on the light transmitted through or reflected by a material depends on the relative orientation of the magnetic field and the light plane of incidence, as shown in Figure 2.

For example, if the magnetic field or the magnetization are aligned perpendicular to the sample plane (XY), which corresponds in reflection to the so called polar Kerr configuration, the dielectric tensor presents the following form:

$$
\varepsilon=\left(\begin{array}{ccc}
\varepsilon & a \Pi & 0 \\
-a \Pi & \varepsilon & 0 \\
0 & 0 & \varepsilon
\end{array}\right),
$$

and therefore the XY plane components of the light EM field are coupled, thus inducing changes in the state of polarization of the light. As a consequence, when linear $p$ polarized ( $s$-polarized) light reflects from a sample, the reflected beam presents a small component of $s$-polarized ( $p$-polarized) light. In other words, the non-diagonal Fresnel 
coefficients accounting for polarization conversion, $r_{p s}$ and $r_{s p}$, depend linearly with $\Pi$. The magnitudes to be experimentally explored are the magnetic field induced rotation, $\theta$, and ellipticity, $\varphi$, experienced by the incident light (see definition in Figure 2), which constitute a complex number denoted in the following the complex Kerr rotation, $\phi$.

A similar effect is observed if the magnetic field or the magnetization is aligned parallel to both the sample plane (XY) and the plane of incidence of the light (XZ), known as the longitudinal Kerr effect.

On the other hand, a different situation occurs if the magnetic field or the magnetization is aligned parallel to the sample plane (XY) but perpendicular to the plane of incidence of the light (XZ). In this case the dielectric tensor becomes:

$$
\varepsilon=\left(\begin{array}{ccc}
\varepsilon & 0 & a \Pi \\
0 & \varepsilon & 0 \\
-a \Pi & 0 & \varepsilon
\end{array}\right) .
$$

In this configuration (known as transversal Kerr or also Voigt configuration for transmission) the magneto-optical element couples components of the EM field that are on the incidence plane, i.e. only the $p$-component of the light will be affected by the applied magnetic field, whereas the $s$-component does not experiment any variation. This means that this configuration involves no change in polarization but a change in the reflected intensity of the $p$-polarized light (see Figure 2).

For the situations regarding transmitted light, i.e. the Faraday effects, the corresponding mathematical expressions are analogous to the ones for the Kerr effects, changing reflectivity by transmission coefficients.

Once the MO effects are introduced, the next step is evidently to find out which materials exhibit optimum MO properties. As a matter of fact, all materials exhibit MO 
activity, but the intensity of this response depends, mainly, on their magnetic nature. Basically, this magnetic nature can be classified according to the magnitude and sign of the magnetic susceptibility $\chi(M=\chi H)$. This is illustrated in Figure 3, where the materials magnetization $M$ versus applied magnetic field $H$ curves are shown for paramagnetic ( $\chi$ small and positive), diamagnetic ( $\chi$ small and negative) and ferromagnetic ( $\chi$ large and positive) materials. Additionally, the magnetic field dependence of $\mathrm{M}$ is also distinctive depending on the magnetic nature of the material. While for ferromagnets the magnetic saturation state may be reached at reasonable small magnetic fields, a linear dependence of the magnetization with magnetic field exists for paramagnets and diamagnets (see Figure 3 (a) and (b)). This differentiation between ferromagnets, with saturation of the magnetization, and paramagnetsdiamagnets, without actual saturation reached in the magnetization, justifies why, when defining the dielectric tensor, the off diagonal components of paramagnets and diamagnets are proportional to the applied magnetic field while those of ferromagnets are proportional to the magnetization. Along with this magnetic classification, the MO constants of ferromagnets happen to be also orders of magnitude larger than those of paramagnetic and diamagnetic materials.

Now, for the specific case of interest of this review, focused on plasmonics, it is necessary to understand the physical origin and magnitude of the MO response of noble metals (the generic plasmonic ones), which actually have a diamagnetic nature, as well as of ferromagnets. In the first case, the optical properties of noble metals are determined in an extended spectral range by the conduction electrons and thus reasonably well described by the Drude model. Therefore their MO constant can be expressed as: ${ }^{[61,62]}$ 


$$
\varepsilon_{m o}=i \frac{\omega_{c}\left(\omega_{p} \tau\right)^{2}}{\omega\left[(1-i \omega \tau)^{2}+\left(\omega_{c} \tau\right)^{2}\right]},
$$

where $\tau$ is the electrons relaxation time; $\omega_{p}$ is the plasma frequency, $\omega_{p}=\sqrt{N e^{2} / \varepsilon_{0} m^{*}}$; and $\omega_{c}$ is the cyclotron frequency, $\omega_{c}=e H / m^{*}$ Here, $e$ corresponds to the electron charge, $N$ to the electronic density, $H$ is the applied magnetic field and $m *$ the electron reduced mass. As it can be easily calculated, for noble metals and for not extremely high magnetic fields, $\omega_{c}$ happens to be much smaller than $\omega_{p}$, and as a natural consequence their resulting MO constants are very small. ${ }^{[63,64,65,66]}$ As a reference, Figure 3 (d) shows the calculated MO constants for Au in the visible under an applied magnetic field of $1 \mathrm{~T}$.

On the other hand, for ferromagnetic metals, the spin-orbit coupling, exchange interaction and the specific band structure of the material determine their MO activity, which happens to be much larger than those of noble metals. ${ }^{[67,68]}$ This is shown in Figure 3 (c), where the spectral dependence in the visible range of the MO constants for Co is also presented. As it can be seen, the values for Co are roughly three orders of magnitude larger than those for Au in the whole spectral range.

But the MO activity is only one of the factors determining the properties of interest in magnetoplasmonic systems. Obviously the optical properties constitute the other factor, and especially the optical losses, which in the end determine the quality of the plasmonic response. In this case, as it is well known, noble metals are characterized for their lower losses and sustain very well defined plasmonic resonances, compared for example to ferromagnetic metals, which usually exhibit large losses and very broad plasmon resonances in the visible range. This is illustrated in Figure 3 (e) and (f) for both propagating and localized plasmon resonances, where the calculated SPP 
propagation distance $\mathrm{e}^{[32,41]}$ and extinction cross section of nanometer-sized metallic particles $^{[38]}$ are plotted (for these calculations, the optical constants for $\mathrm{Au}$ and $\mathrm{Ag}$ were taken from Ref. [69], and those for ferromagnetic metals from Ref. [70]). As it can be seen, the SPP propagation distance is much larger for noble than for ferromagnetic metals and the LSP resonances are narrower because of the optical losses of the last ones.

Therefore, summarizing all these previous concepts in a few words, when dealing only with noble metals, they constitute excellent plasmonic systems with very small MO effects. On the other hand, ferromagnetic metals are paradigmatic in terms of their sizeable MO effects but lack of competitive plasmonic properties. The initial ansatz then is that an optimum combination of both MO and plasmonic characteristics can be obtained by mixing ferromagnets and noble metals. The ferromagnet endorses the MO activity to the system, whereas the noble metal allows SP excitation with low optical losses. Actually, this approach has been followed up by a number of different groups using either metallic or dielectric ferromagnetic materials. In these novel structures, commonly denoted as magnetoplasmonic systems, magnetic and plasmonic properties are intertwined, allowing for example plasmonic properties to become tunable upon the application of a magnetic field (active plasmonics), ${ }^{[37]}$ or the MO effects to be largely increased by plasmon resonance excitation, as a consequence of the enhancement of the EM field in the MO active component of the structure. ${ }^{[71]}$ In this last case, the study of the enhanced MO activity in structures with subwavelength dimensions is especially interesting since they may be viewed as nanoantennas in the visible range with MO functionalities. The light harvesting properties of these systems upon plasmon resonance excitation bring as a consequence an enhanced EM field in its interior, and in particular in the region where the MO active component is present. Moreover, 
magnetoplasmonic systems have opened new routes for the development of higher performance gas and biosensing platforms ${ }^{[36,72,73,74]}$ as well as the exploitation of nonreciprocal effects in devices with potential applications in the telecommunications area. $^{[75]}$

With all this in mind, in this review we will mainly focus on the intertwined effects that plasmonics and magneto-optics offer from the fundamental and applied viewpoints. We will make special emphasis on the enhancement of the MO activity of the structures due to plasmon excitation, considering the interest of these systems for the search of materials with strong MO activity, and on the magnetic field control of the plasmon properties thanks to the presence of a ferromagnetic component that are relevant for the obtention of active plasmonic configurations. Additionally, there are in the literature a number of relevant works dealing with exciting phenomena that are not directly related to those described before, but which are as well associated with nanostructures where plasmonic and magnetic features coexist, and which also make use of the "magnetoplasmonics" term. These studies will be also briefly described in a specific section as they constitute, together with the results presented in the rest of the review, a global vision on the phenomenology associated to systems which share magnetic and plasmonic functionalities.

Therefore, the present manuscript is organized as follows: in section 2 we will detail the effects that plasmon excitation (either localized or propagating) have on the MO activity of different kinds of structures. Section 3 will be devoted to review the works focused on the magnetic field effects on the plasmon properties of different kinds of systems. We will complete this work in section 4 with some examples of actual and potential applications of the described phenomena. The variety of studies considering systems with independent magnetic and plasmonic properties will be briefly put forward in 
section 5. Finally in section 6 we will present our conclusions and the perspectives that, we believe, this whole phenomenology offers for the next years.

\section{Plasmon resonance effects on the MO activity}

The MO activity of a system is the result of the combined effects of the pure optical and MO properties of the global structure. For example, as it can be seen in Figure 2, the complex Kerr rotation, $\phi$, in polar configuration is defined as:

$$
\phi=\theta+i \varphi=r_{p s} / r_{p p}
$$

Therefore, both an increase in $r_{p s}$ (polarization conversion or pure MO contribution) or a reduction in $r_{p p}$ (pure optical contribution) are mechanisms to enhance the MO activity, and the excitation of plasmon resonances is a very effective way to affect both the pure optical and pure MO parameters as we will show in what follows.

Regarding the pure MO contribution, let us consider the case of a nanostructure (either continuous thin film or nanoparticle) with a very thin layer of MO material of thickness $d$. In this system, the MO polarization conversion, $r_{p s}$, can be expressed as: ${ }^{[18,76,77,78,79]}$

$$
\left|r_{p s}\right| \propto\left\langle E_{p} E_{s}\right\rangle d\left|\varepsilon_{m o}\right|,
$$

where $\left\langle E_{p} E_{s}\right\rangle$ is the mean value of the product of both components of the field inside the MO layer, normalized to the incoming intensity, and $\varepsilon_{m o}$ its MO constant. As it can be seen, the polarization conversion or pure MO contribution is directly proportional, among other factors, to the EM field inside the MO material. Being EM field 
enhancement one of the main properties of plasmon resonances excitation, it can be readily concluded that they can induce an increase in the MO activity.

On the other hand, the excitation of plasmon resonances usually induce minima in the magnitudes corresponding to the pure optical contribution as for example is the case in the excitation of SPPs using a Kretschmann (or attenuated total internal reflection, ATR) configuration, where a minimum in the reflectivity is the signature of plasmon excitation. In this specific case, the minimum value of the pure optical contribution, $r_{p p}$, has as a direct consequence a maximum in the Kerr rotation.

Therefore, it is possible to devise a system which exhibits an enhanced MO activity either by largely concentrating the EM field at the MO active layer (pure MO effect), or by reducing the reflectivity of the system (pure optical effect), or very often by the combination of both. In what follows, we will detail the above described phenomenology in systems sustaining both localized and propagating surface plasmons, discussing which effect prevails in each situation. In particular, for LSPs, where strong nanoantenna effects (enhancement of the EM field) take place, the influence of the MO part is more important, while for SPPs the optical part dominates as usually the propagating plasmon excitation gives rise to a reflectivity reduction.

\subsection{Systems with localized surface plasmons}

Back in 1987, Hui and Stroud already predicted large Faraday coefficients in suspensions of small Faraday-active nanoparticles embedded in a host at the surface

plasmon frequency of the particles, ${ }^{[80,61]}$ with a physical origin for this effect attributed to the increased local field within or just outside the nanoparticle. Motivated by these theoretical works, already in 1988 Yusuf et al. explored the MO activity of $\mathrm{Fe}_{3} \mathrm{O}_{4}$ magnetic particles diluted in a fluid finding a large enhancement around 500nm. ${ }^{[81]}$ They interpreted their results according to Hui and Stroud's calculations, attributing the 
enhancement to the presence of a resonance in the small nanoparticles, even though this resonance cannot be of plasmonic nature since $\mathrm{Fe}_{3} \mathrm{O}_{4}$ is non-metallic.

In these first theoretical studies both the $\mathrm{MO}$ and the plasmonic properties were endorsed by the same component, modeled by a Drude dielectric tensor. Subsequently, the plasmon resonance effects on the MO activity of many different real materials have been studied, including nanoparticles made exclusively from ferromagnetic (FM) metals but with no purely plasmonic component, nanoparticles combining ferromagnets and noble metals or, as an alternative to this last one, noble metal nanoparticles embedded or on top of a ferromagnetic dielectric matrix. The main results for all these kinds of systems are described in what follows according to this classification.

\subsubsection{FM metal nanoparticles}

The chronological and conceptual continuation of the pioneering works of Hui et al. was realized by measuring Polar Kerr and Faraday rotation spectra in a number of metal-dielectric granular films for different ferromagnetic metals such as Co-Fe or Co, grown using deposition techniques such as sputtering and e-beam evaporation. ${ }^{[82,83,84]}$ These works have shown that the MO properties depend on the volume fraction of ferromagnetic metal, and they also found singularities which have been related to plasmon excitation in the granular films. More recently, a purely ferromagnetic system studied in more detail and where the localized plasmon excitation effects on the MO activity has been demonstrated consists of arrays of $\mathrm{Ni}$ nanowires obtained by electrochemical infiltration of $\mathrm{Ni}$ inside the pores of anodized alumina membranes. ${ }^{[85,86,87]}$ As it can be seen in Figure 4 (a), and despite the fact that the amount of $\mathrm{Ni}$ in the nanowires sample is only $17 \%$ of that of a continuous film, the observed Kerr rotation around $3.2 \mathrm{eV}$ is very similar for both cases, whereas in the infrared region the Kerr rotation is basically proportional to the amount of $\mathrm{Ni}$. This 
behaviour has been attributed to the excitation of LSP resonances in the Ni nanowires. This excitation induces an enhancement of the EM field inside the nanowires that gives rise to an increase in the polarization conversion ratio $r_{p s}$ in the spectral region where the LSP resonance peak appears. By analyzing the dependence of the MO activity on the Ni wires diameter, and taking also into account the effect of variations of the matrix refractive index, it was concluded that the excitation of LSPs in the Ni wires was affecting the MO response. To illustrate this, Figure 4 (b) shows calculated results of the effect that the different components that appear in the MO activity have on the overall MO response of the nanowire system. We first show the modulus of the wires MO activity (Kerr complex rotation in particular) normalized to that of a continuous film for three different wire diameters with the same amount of $\mathrm{Ni}$. As it can be observed, and despite the fact that the amount of $\mathrm{Ni}$ in the nanowires samples is about $1 / 6^{\text {th }}$ of that of a continuous film with equivalent thickness, the magnitude of the MO activity is comparable to that of the continuous film with a peak whose spectral position varies with the wires diameter in the same way that the LSP of a metallic wire, that is, the peak is red-shifted for larger diameters. ${ }^{[38]}$ Moreover, taking into account the spectral shape of the nanowires reflectivity coefficients $r_{p s}$ and $r_{p p}$ normalized to the values for a continuous film, it can be concluded that $r_{p s}$ is in this case the main responsible of the observed enhancement in the LSP region, while $r_{p p}$ gives an almost wavelength independent contribution.

Like in the nanowires case, Ni nanodisks also exhibit LSP resonance effects on their MO properties. ${ }^{[8,89]}$ In this last work, Ni nanodisks of different diameters have been studied, which present a surface plasmon resonance excitation whose spectral position depends on the disk size (see insets in Figure 5). Surprisingly, in this work it has been shown that the sign of longitudinal Kerr rotation loops depends on whether they are 
taken at wavelengths higher or lower than the LSP resonance, as shown in Figure 5.

Due to the relationship between the Kerr rotation and the polarizability, ${ }^{[90]}$ this behavior is attributed to the fact that the real part of the particle polarizability changes its sign at the LSP resonance, and this change of sign is transferred to the MO response. In a simplified picture, this can be understood as follows: the electrons of the nanoparticle oscillate in phase (out of phase) with the exciting electric field for energies below (above) the resonance, and therefore the Lorentz force and as a consequence the MO response change sign.

Additionally, introducing order in these kind of systems gives rise to so-called magnetoplasmonic crystals, in which the effect of the associated lattice anomalies (Wood, Rayleigh, ...) on the MO response has been recently analyzed. ${ }^{[91]}$

\subsubsection{Noble metal-FM nanoparticles}

Localized surface plasmon related effects on the MO activity have also been reported for nanosystems where both ferromagnetic and plasmonic components coexist, mainly in noble metal-ferromagnetic nanoparticles and nanodisks. A first step was given in 2005 by Li et al., who studied the Faraday response of colloidal solutions of dumbbelllike Ag-CoFe $\mathrm{O}_{4}$ nanoparticles. ${ }^{[92]}$ They found a different Faraday response for $\mathrm{CoFe}_{2} \mathrm{O}_{4}$ nanoparticle and $\mathrm{Ag}-\mathrm{CoFe}_{3} \mathrm{O}_{4}$ dimer solutions, and attributed this difference to the plasmonic contribution of Ag nanoparticles. More recently, clear surface plasmon resonance enhancement of the MO activity have been observed in $\mathrm{Au} / \mathrm{Co} / \mathrm{Au}$ nanosandwiches obtained by colloidal lithography (Figure 6 (a) and (b)). ${ }^{[93]}$ In this work, the diameter of the nanodisk sandwiches was varied between 60 and $110 \mathrm{~nm}$, while maintaining the thickness of the metallic layers. The change of the nanodisk aspect ratio is directly translated to the position of the LSP resonance, varying in the 2.2 $\mathrm{eV}$ region as can be seen in Figure 6 (a), with a red shift in the peak position as the 
diameter of the nanodisks increases. Similar red shift trend is observed in the features present in the corresponding polar complex Kerr rotation spectra, in this case centered around $1.9 \mathrm{eV}$ (Figure 6 (b)). Theoretical calculations confirm and quantitatively reproduce the experimentally observed results, being interpreted in terms of an enhancement of the EM field in the nanodisk upon LSP resonance excitation. Similar enhancement has also been reported for different types of noble metal-ferromagnetic nanoparticle systems, such as colloidal gold-magnetite nanoparticle composite films ${ }^{\text {[94] }}$ or gold coated maghemite nanoparticles (Figure 6 (c) and (d)). ${ }^{[95,96]}$ In this kind of noble metal-ferromagnetic oxide systems, the spectral and spatial overlap of the LSP resonance with electronic transitions involved in the MO spectral response may play a relevant role. As it can be seen in Figure 6 (c) and (d), an enhanced Faraday rotation in the spectral range where both plasmon resonance and intrinsic electronic transitions of the ferromagnetic oxide coexist has been observed. ${ }^{[95]}$ A correlation between the MO activity and the excitation of plasmon resonances has also been recently observed in core-shell Au-Ferrite nanowires. ${ }^{[97]}$ Additionally, purely metallic systems like AgFe alloy nanoparticles, ${ }^{[98]}$ or core-shell Co-Ag nanoparticles have also been studied. ${ }^{[99]}$ In this last work, Wang et al. clearly demonstrate, both theoretical and experimentally, the direct relation between optical absorption and MO activity in their Co-Ag nanoparticles. As it can be seen in Figure 6 (e) and (f) for different nanoparticle stoichiometries, similar trends with composition are observed in both the absorbance and Faraday rotation, with a strong maximum around $3.2 \mathrm{eV}$, and higher values of absorbance and Faraday rotation for the particles with higher Ag concentration.

Once the plasmon resonance effects on the MO activity of these systems are generally understood, different aspects that could influence this effect have been considered. For example, Du et al. have studied size and shape effects on $\mathrm{Au} / \mathrm{CoPt} / \mathrm{Au}$ nanodisks 
obtained by physical methods, ${ }^{[100,101,102]}$ finding a large tuning ability of the MO enhancement with the geometrical parameters of the plasmonic entities. Additionally, it is important to keep in mind that in these kinds of MO studies, experiments are usually performed in ensembles of $10^{8}-10^{9}$ nanodisks or nanoparticles, and if they are close enough to each other, EM interaction effects may play a relevant role. This has been analyzed by Demidenko et al., who have studied theoretically local-field effects in the magnetoplasmonic response of nanodisks deposited on a dielectric substrate, ${ }^{[103]}$ confirming that both interparticle and particle-substrate interactions have to be taken into account to describe the magneto-optical properties of the system.

Moreover, to obtain an enhancement in the MO activity in this kind of systems, it is not really necessary that the MO material and the LSP sustaining component are in contact, as far as the EM field associated to the resonant plasmonic entity reaches the MO material. This has been demonstrated in structures consisting of an array of gold nanodisks separated from a $\mathrm{Au} / \mathrm{Co} / \mathrm{Au}$ trilayer by a $\mathrm{SiO}_{2}$ layer. ${ }^{[104]}$ The $\mathrm{LSP}$ sustained by the Au nanodisks modifies the MO response of the continuous trilayer due to the redistribution of the EM field in the whole structure. In fact, depending on the specific spectral range, the EM field intensity inside the Co layer may be enhanced or even reduced, and it has been indeed shown that the spectral dependence of the pure MO component, $r_{p s}$, is strongly correlated to the EM field value inside the MO active layer. Besides, in this structure both $r_{p p}$ and $r_{p s}$ contribute similarly to the modification of the MO response.

Interestingly enough, plasmon resonance effects are so strong that it has become possible measuring a sizeable MO activity in diamagnetic Au nanostructures. ${ }^{[90,105,106]}$ This effect can again be explained in terms of the particle polarizability, which diverges at the resonance peak, thus maximizing the Lorentz force on the collective response of 
the electrons and therefore the MO response at the LSP position. Paying attention to the spectral shape of the MO activity, in purely plasmonic systems is different from that of noble metal-ferromagnetic systems. This is related to the predominance of the imaginary part of the off-diagonal elements of the dielectric tensor in the magnetooptical response for purely plasmonic systems, while on the metal-ferromagnetic case it is dominated by the real part, as has been shown by studying the effect that the amount of ferromagnetic metal in the systems has on the spectral dependence of the MO effect. $^{[107]}$

In all the works described so far, the main goal was the study of ways to enhance the MO activity of the systems, qualitatively due to the enhanced EM field at the nanostructure upon plasmon excitation. A more quantitative relationship was directly shown by Wang et al. ${ }^{[99]}$ who correlate the MO activity of their core-shell Co-Ag nanoparticles with the amount of EM field (actually the electric field) within the Co core of the nanoparticles as a function of the Co amount (Figure 7 (a)). Moreover, even though all these structures have dimensions smaller than the wavelength corresponding to plasmon excitation, the EM field distribution within these nanostructures does not necessarily need to be homogeneous (see Figure 7 (b)). Actually, the direct relation of the MO activity with the EM field intensity (see Eq. (6)) can be used to experimentally determine the EM field distribution inside the resonant magnetoplasmonic nanostructure. In this way, the MO active component can be used as an internal EM field probe, for example by tuning the position of a Co layer within a Au nanodisk. In Figure 7 (c) we show results from Meneses et al., ${ }^{[79]}$ who have demonstrated that it is indeed possible to determine the internal distribution of the EM field inside $\mathrm{Au} / \mathrm{Co} / \mathrm{Au}$ nanodisks by recording the dependence of the MO activity with the Co layer position. They grew $\mathrm{Au} / \mathrm{Co} / \mathrm{Au}$ trilayered structures changing the position of the Co layer within 
the system, both in thin film and in nanodisk form. By evaluating the MO activity versus Co position in both systems, it is clearly observable how it exhibits the usual exponential decay in the case of a continuous film, while presenting maximum values at the top and bottom interfaces in the case of the nanodisks. Theoretical calculations of the EM field distribution for both cases have a very good quantitative agreement with the experiments.

As mentioned before, the EM field distribution within a resonant nanodisk is not homogeneous, and therefore ways to boost this redistribution, concentrating the EM field in the MO active materials while simultaneously reducing it in the non MO active, lossy ones is of obvious interest. Routes that allow engineering the EM field distribution within a metallic nanostructure include for example the insertion of a dielectric layer, giving rise to the appearance of multiple mode resonances that come along with a strong redistribution of the EM field. This has previously been shown in gold-silica-gold nanodisks, ${ }^{[108,109,110]}$ whose results can easily be explained according to the so called plasmon hybridization model. ${ }^{[111]}$ Regarding magnetoplasmonic structures, onion-like nanoparticles have been studied by Abe et al. ${ }^{[112,113,114]}$ from a theoretical point of view, showing that multiple mode resonances can also be observed in this kind of systems, and predicting an enhancement of the MO activity associated to the excitation of plasmon resonances. Recently, simultaneous large MO activity and low optical losses have been found in $\mathrm{Au} / \mathrm{Co} / \mathrm{Au}-\mathrm{SiO}_{2}$ nanodisk structures, ${ }^{[115,116]}$ shown in Figure 8. In these systems, fabricated by hole mask colloidal lithography, ${ }^{[117]}$ Banthí et al. compare the optical extinction and MO activity of such structures when a $20 \mathrm{~nm}$ thick $\mathrm{SiO}_{2}$ layer is placed below and above the Co layer, as well as for a pure $\mathrm{Au} / \mathrm{Co} / \mathrm{Au}$ nanodisk system. The insertion of the dielectric layer produces the splitting of the single resonance into two modes, at low and high wavelengths, which manifests both in the 
optical as well as on the MO spectra. While the MO spectra for both metal-dielectric systems are basically unaltered, with a shoulder in the spectral region corresponding to the MO peak of the fully metallic structure, and a stronger peak at larger wavelengths (see Figure 8 (c) and (d)), the extinction spectra strongly change depending on the position of the $\mathrm{SiO}_{2}$ layer (Figure 8 (a) and (b)). Actually, for the system with the dielectric layer below the Co, the extinction spectrum has a similar shape than the MO one, with a peak at high wavelengths and a shoulder at low wavelengths. However, if the dielectric layer is placed above the Co, the intensity of the optical features reverses, the low wavelength peak being now stronger that the high wavelength one. This can be explained considering the EM field distribution for the two resonance wavelengths and both dielectric layer positions (insets of Figure 8 (a) and (b)). As it can be observed, in all cases the EM field concentrates in the metallic regions, being maximum in the pure Au nanodisk layers (this determines the strength of the extinction peaks). Regarding the EM field at the MO active component (the Co layer), it is similar for both positions of the dielectric layer (explaining the similar MO activities). As a consequence, for the configuration where the dielectric layer is placed above the Co one, it is possible to simultaneously obtain a situation with high MO activity and low optical losses, namely in the high wavelength region (Figure 8 (b) and (d)), with a figure of merit MO activity versus optical losses much larger than that of the corresponding fully metallic nanodisks. ${ }^{[115]}$

\subsubsection{Noble metal nanoparticles and FM dielectric layers}

An alternative to deal with systems where both plasmon resonances and MO activity components have a nanoparticle-like nature is to fabricate structures where both functionalities are provided by structurally different elements. We have previously

described the case of a $\mathrm{Au}$ nanodisk- $\mathrm{Au} / \mathrm{Co} / \mathrm{Au}$ thin film trilayer system, ${ }^{[104]}$ which 
needed a dielectric spacer between the nanodisks and the trilayer to preserve the strong nanodisk plasmon resonances. However, a simpler structural scenario may be faced if the MO component itself is dielectric in nature. In this line, a number of works study systems where the plasmonic activity lies in noble metal nanoparticles, whereas the MO part is provided by continuous layers or matrices of ferromagnetic materials which, to maintain the localized nature of the noble metal nanoparticle plasmon, are dielectric. Moreover, the low optical absorption that a ferromagnetic dielectric matrix in principle exhibits is a potential advantage. In this sense, in 2006 Tomita et al. ${ }^{[118]}$ studied layers of $\mathrm{Au}$ nanoparticles embedded in garnet matrices obtained by co-sputtering together with thermal annealing. They observed a change in the sign of the Faraday rotation in the spectral region of the LSP of the Au nanoparticles and attributed it to a possible coupling between the MO Kerr effect of garnet and the LSP. Later, other groups studied similar systems observing an increase of the Faraday rotation in the spectral region of the LSP resonance. ${ }^{[119,120,121,122,123,124]}$ For example, Figure 9 shows the case of Au nanoparticles embedded in a garnet layer, where the nanoparticles were produced by annealing of a very thin Au layer. As the annealing temperature increases, the shape of the particles is better defined and the size distribution narrows, giving rise to a sharper LSP resonance (Figure 9 (a)) and therefore to a stronger enhancement of the Faraday signal (Figure 9 (b)). Moreover, a correlated blue-shift is observed in both optical and MO magnitudes when the annealing temperature is increased. ${ }^{[120]}$

\subsection{Systems with propagating surface plasmons}

The opportunity of MO activity enhancement by exploiting plasmon resonances was already foreseen in the '70s, short after the excitation of SPPs by means of ATR configuration was proposed: Ferguson et $a .^{[125,126]}$ used this configuration (Kretschmann geometry) to excite SPPs in thin ferromagnetic films (Fe or Ni) deposited 
on top of a glass prism and theoretically predicted an increase in the transversal MO response of the system. Indeed, the enhancement in the MO activity introduced by the excitation of SPPs in the transversal configuration is as high as to allow a comfortable experimental determination of the minute MO constants of several noble metals. ${ }^{[66]}$ Since then, an ample number of systems, composed not only of continuous but also of patterned thin films, have been explored for this purpose. In this last direction, perforated continuous plasmonic films are known to present exciting phenomena related with extraordinary transmission properties, ${ }^{[127]}$ and recently magnetoplasmonic systems with this phenomenology have been also studied. In what follows we will describe the main published works dealing both with systems exhibiting SPPs and extraordinary transmission like properties.

\subsubsection{Continuous layers}

During the late nineties and the beginning of this century, Safarov, Hermann and coworkers pioneered investigations involving noble metal-ferromagnetic metal multilayered systems, ${ }^{[17,18,76]}$ with SPPs excited using the mentioned ATR geometry (see sketch in Figure 10 (a)). These works analyzed the effect of SPP excitation on the MO response in $\mathrm{Au}-\mathrm{Co}$ multilayered systems using different magnetic configurations (polar, transversal and longitudinal Kerr configurations). They reported an enhancement of the MO response due to SPP excitation and a strong relationship between MO activity and EM distribution inside the Co layer.

In ATR geometry, the plasmon excitation brings as a consequence a resonance shape of the angular dependent reflectivity, with a minimum at a specific incidence angle. This is in contrast with the case when no SPP is excited, where the reflectivity angular dependence is featureless (see Figure 10 (b)). The same behavior is observed for the MO signal, with a strong increase when the SPP is excited (Figure 10 (c)). In fact, the 
main reason for the observed MO activity enhancement is the reduction of the reflectivity, even though the MO component is also incremented, due to the enhancement of the EM field inside the MO active layer. This last aspect is shown in Figure 10 (d), where an angle independent exponential decay is observed in the EM field in the absence of SPP excitation, while a strong increase of the EM field at the MO active layer is observed for specific incident angles under SPP excitation. In the particular case of transversal geometry, an important contribution to the MO activity comes from the magnetic field wavevector modulation of the SPP. This modulation will be discussed in detail in section 3 .

Other groups have considered the analysis of these systems from a theoretical point of view, ${ }^{[128,129,130,77]}$ showing that the optimum structures to maximize the MO response depend on the specific measurement configuration (either polar, transversal, etc...). For example, Bonod et al. ${ }^{[77]}$ have shown how in transversal configuration the optimization is achieved by optimizing the plasmon excitation, whereas in longitudinal or polar configurations, optimizing the magnetic layer may be better.

More recently, a deeper analysis of this kind of systems has been carried out, focused mainly in the transversal configuration, where the plasmon excitation, ${ }^{[131]}$ the dependence on the ferromagnetic layer thickness ${ }^{[132]}$ and its position within the layer, ${ }^{[133,134,78]}$ the nature of the plasmonic material, ${ }^{[135,136]}$ the crystalline and interface quality, ${ }^{[137]}$ have been studied in a series of noble metal/ferromagnetic/noble metal trilayer structures or nanocomposite ${ }^{[138,139]}$ thin films. For example, Figure 11 shows how both the reflectivity (which is related to the SPP excitation quality) and the purely MO component, $\Delta R_{p p}$ here, can be optimized by appropriate choice of the layer thicknesses to obtain the desired MO enhancement. The optimization of each component is obtained with different thicknesses, and a compromise has to be 
established depending on which magnitude, $R, \Delta R_{p p}$ or $\Delta R_{p p} / R$, we are interested in maximizing. ${ }^{[78]}$

In the preceding paragraphs, we have presented works in which Kretschmann geometry is used to excite SPPs, but this can also be achieved by the use of periodic systems such as gratings. Following this approach, Newman et al. ${ }^{[140]}$ and Grunnin et al. ${ }^{[141]}$ have studied the effect of such excitation in $\mathrm{Ni}$ and Co gratings as well as $\mathrm{Ni}$ gratings covered by $\mathrm{Ag}$ thin films, and Clavero et $a .^{[142]}$ analyze $\mathrm{Au} / \mathrm{Co} / \mathrm{Au}$ gratings, finding an enhancement of the MO activity correlated with the increase of the EM field associated to the presence of SPPs in the system.

As previously mentioned, the use of MO materials of dielectric character offers the advantage over ferromagnetic metals of the lower optical absorption, which makes continuous films systems composed of noble metal layers and MO dielectric layers very interesting. In this sense, a preliminary experiment on the influence of surface plasmons on the Faraday effect in bismuth-containing iron garnet (Bi-IG) films coated with a thin film of Au was presented by Bremer et al. in ATR geometry. ${ }^{[143]}$ On the other hand, Kochergin et al. ${ }^{[144]}$ detected an enhancement in the longitudinal MO signal associated with the propagation of a SPP for a Bi-IG grating coated with a $\mathrm{Ag} / \mathrm{Au}$ multilayer, and subsequently proposed the use of SPP excitation as a tool for resolution increasing in MO imaging of films. ${ }^{[145]}$

Finally, mention that the strong concentration of EM field generated by the SPP excitation has effects not only on the linear component of the MO effects, but also on the MO second-harmonic generation, as demonstrated by Newman et al. for $\mathrm{Ni}$ gratings,${ }^{[146]}$ by Pavlov et al. in ultrathin $\mathrm{Au} / \mathrm{Co} / \mathrm{Au}$ multilayers, ${ }^{[147]}$ and more recently by Valev et al. in chiral Ni nanostructures. ${ }^{[148]}$ 


\subsubsection{Perforated continuous layers and extraordinary transmission effects}

An alternative route to excite surface plasmons in continuous films is to fabricate arrays of holes in them, obtaining nanoperforated structures which are also called plasmonic crystals. ${ }^{[149]}$ This kind of systems is nowadays object of intense investigation, since they may present extraordinary optical transmission properties. ${ }^{[127]}$ Regarding the specific scope of this review, even though not in transmission but in reflection, the effect of SPP excitation on the MO activity has been reported in hexagonal arrays of subwavelength holes in optically thin cobalt, ${ }^{[150,151]}$ iron, ${ }^{[152,153,30]}$ and nickel membranes, ${ }^{[154,155]}$ as well as in nanocorrugated films. ${ }^{[156,157,158,159,160,161,162]}$ The excitation of propagating plasmons due to the extra momentum provided by the underlying periodic structure gives rise to clear signatures in the reflectivity of the samples, in a similar fashion as in the ATR configuration. This plasmon excitation influences the MO response, leading to an enhancement. This is clearly seen in Figure 12, where results for a perforated iron membrane with hexagonal symmetry are presented. Figure 12 (a) shows a sketch and SEM image of such membrane, with indication of the membrane lattice parameters. Then, in Figure 12 (b) the correlation between the reflectivity minima and the maxima in the polar Kerr rotation at normal incidence is presented, highlighting the importance of the optical component in the increase of the MO activity. Figure 12 (c) presents the effect on the TMOKE signal. Here, both the angle of incidence and the sample azimuthal angle are varied to show that the spectral location of the enhancement follows the SPP lattice band structure. This kind of systems may also sustain localized surface plasmons in the holes, which can also influence the MO response, as is discussed in Ref. [154].

More directly dealing with extraordinary transmission kind of systems and phenomena, the effect that a magnetic field has on the optical transmission through nanostructured 
conducting films was first analyzed by Strelniker and Bergman ${ }^{[163,164]}$ for a Drude-like metal in the presence of a magnetic field applied in the plane of the sample. They found that the frequency of the extraordinary transmission peaks depends on both the magnitude and direction of the applied magnetic field. Also, the tuning of the extraordinary optical transmission through subwavelength hole arrays in Ag films by an external magnetic field has been calculated. ${ }^{[165]}$ Obviously, as no ferromagnetic material is present in these structures, the predicted effects are very small unless very large magnetic fields (tens of Tesla) are applied.

On the other hand, regarding noble metal-garnet systems, Belotelov et al. analyzed the effect that the extraordinary transmission has on the Faraday and Kerr effects of nanostructured $\mathrm{Au}$ thin films deposited on top of a garnet layer, both in $2 \mathrm{D}^{[166]}$ and $1 \mathrm{D}$ perforated systems ${ }^{[167,168,27]}$, taking into account all the different MO configurations: polar, ${ }^{[166]}$ longitudinal ${ }^{[167]}$ and transversal. ${ }^{[168,27]}$ Large enhancements of the MO effect were predicted at the transmission peaks, as shown in Figure $\mathbf{1 3}$ (a) and (b). In particular, Figure 13 (a) corresponds to the calculated wavelength dependence of the transmittance and the Faraday rotation for a perforated Au film deposited on top of a magnetic dielectric Bi-IG film of a specific thickness $(h=118 \mathrm{~nm})$ in polar configuration. A correlation between a transmission resonance and a maximum in the Faraday rotation for wavelength $963 \mathrm{~nm}$ is clearly observed. Figure 13 (b) presents the evolution of both the transmittance and the Faraday rotation with the thickness of the magnetic dielectric film $h$ at this wavelength, showing that the maximum enhancement of Faraday rotation takes place at $h=118 \mathrm{~nm}$. Moreover, this graph also indicates that transmission resonances and enhanced Faraday rotation are not fully correlated, as an effective TM ( $p$-polarized)-TE ( $s$-polarized) polarization conversion also requires that the edges of the photonic bandgaps for both TE and TM modes coincide, which is only 
achieved for appropriate values of $h{ }^{[166]}$ Wurtz et al. experimentally analyzed a similar type of system, ${ }^{[169]}$ and Figure 13 (c) shows the effect of a perpendicularly applied magnetic field on the cross-polarized intensity transmitted through a perforated Au/garnet structure. As it can be seen, the polarization rotation increases with the magnetic field intensity at the crystal band edges. Finally, mention that Khanikaev et $a l .{ }^{[26]}$ have also predicted large Faraday rotations at frequencies near extraordinary optical transmission in perforated optically thick metallic films with holes filled with a MO active material.

To summarize all this section, the effect of plasmon resonance excitation on the MO activity of magnetoplasmonic system has been clearly demonstrated in multiple systems. In general, since the MO activity depends both on the magneto-optic and optical contributions, either one of them, or even both, may affect the overall result. For example, we have shown cases where the optical part dominates the global MO activity (SPP excitation in ATR configuration), and situations where both contributions are of similar importance (systems with LSPs in close proximity to MO active layers). Besides, the direct relation between the pure MO component and the EM field intensity in the MO active layer has been established.

Finally, we would like to point out that the increase in the magneto-optical effect due to plasmon excitation can have an interesting derivative in the so-called inverse Faraday effect, which consists on the generation of a permanent magnetic field induced by incident circularly polarized light. ${ }^{[170,171]}$ The intensity of this effect is very small, and so far it has been demonstrated by using high power femtosecond lasers. ${ }^{[172,56]}$ Some recent theoretical results show that the EM field enhancement associated with plasmonic resonances could help increasing this effect. ${ }^{[173,174,175,176]}$ 


\section{Magnetic field effects on the plasmonic properties}

The effects of an external magnetic field on plasmon resonances may be observed both in systems presenting localized and propagating surface plasmons. In the first case, and for metals whose optical and MO properties are well described by a Drude model with low optical losses, a splitting of the plasmon resonance under very large magnetic fields has been predicted in polar configuration and under circularly polarized light illumination $^{[177]}$ (see sketch in Figure 14). However, these effects are extremely small to be realistically observed in an actual system. Much clearer effects have been predicted and observed in systems exhibiting propagating plasmons, which will be exposed in this section.

The theory of the effect of a magnetic field on a surface plasmon polariton (SPP) was developed in the early seventies, together with the formalism to explain the propagation of these surface waves in different kinds of materials, including anisotropic ones. Initially, the work was focused on degenerate semiconductors, such as n-type InSb. For these materials the plasma frequency is comparable to the cyclotron frequency at magnetic fields of the order of a few Tesla, and therefore the MO constants can be varied from zero to values comparable to those of the optical ones (see Eq. (4)) and the magnetic field induced effects are quite strong. In these works, ${ }_{[1,2,3,178,179,180,181]}$ all possible magnetic configurations were analyzed for the most basic geometry of an interface between two semi-infinite media. These studies already contain the main phenomenology regarding magnetic field induced effects in SPP waves. In particular, when the magnetic field is applied either perpendicular to the surface or parallel to the SPP wavevector (polar and longitudinal configurations), it induces a TE component in the initially pure TM surface wave, and introduces a term quadratic with the magnetic term $\Pi$ (see Eq.(1)) in the SPP dispersion relation, as it can be seen in Figure 14. On the 
other hand, a magnetic field applied in the interface plane and perpendicular to the SPP wavevector (transversal or Voigt configuration) induces a term linear with Пin the SPP dispersion relation, and it does not produce any modification of the TM character of the surface wave. In this last case, the SPP wavevector, $k_{s p}$, can be expressed as:

$$
k_{s p}(\Pi)=k_{s p}^{0}+\Delta k_{s p}(\Pi)
$$

with $k_{s p}^{0}$ being the SPP wavevector of the single interface ${ }^{[31]}$ without applied magnetic field and $\Delta k_{s p}$ being the magnetic field induced modulation of the surface plasmon wavevector. For an interface composed of a non-magnetic dielectric material of dielectric constant $\varepsilon_{d}$ and a magnetic metal of diagonal dielectric constant $\varepsilon_{m}$ and MO component $\varepsilon_{m o}$, this SPP wavevector modulation can be expressed as:

$$
\Delta k_{s p}=\frac{-k_{s p}^{0} \varepsilon_{m o}}{\sqrt{\varepsilon_{d} \varepsilon_{m}}\left(1-\frac{\varepsilon_{m}^{2}}{\varepsilon_{d}^{2}}\right)}=\frac{-k_{0} \varepsilon_{d}^{2} \varepsilon_{m o}}{\left(\varepsilon_{d}+\varepsilon_{m}\right)^{3 / 2}\left(\varepsilon_{d}-\varepsilon_{m}\right)},
$$

where $k_{0}$ is the light wavevector in vacuum. ${ }^{[182]}$ In later years, the case of $\mathrm{Ni}$ and degenerate semiconductors thin films embedded between two semi-infinite dielectrics $^{[183,184,185,186,187,188,189]}$ or even multilayers ${ }^{[190]}$ was also analyzed, showing similar behaviour.

Recently, with the push of plasmonics, there has been a renewed interest of the effect of the magnetic field on SPPs, now focused on SPPs supported by metals. For these systems, the most attractive configuration is that with the magnetic field applied parallel to the interface and perpendicular to the SPP wavevector, where the TM nature of the 
plasmon is preserved, the SPP wavevector is modified and a magnetic field induced non-reciprocity effect appears $(\omega(\boldsymbol{k}) \neq \omega(-\boldsymbol{k}))$, an effect that will be specifically discussed later in this section.

The wavevector modification in pure ferromagnetic layers and in a single interface case has been studied using two gratings to induce the light-SPP conversion and viceversa. ${ }^{[191]}$ However, the strong optical losses associated with the ferromagnetic metals and the resulting short propagating distance of the surface plasmons makes these materials not interesting from an applied point of view. To overcome this limitation, again the combination of noble metal and ferromagnetic layers allows facing this problem, and for example $\mathrm{Au} / \mathrm{Co} / \mathrm{Au}$ structures have been studied. Temnov et al. have explored the wavevector modulation using plasmonic microinterferometers, as shown in Figure 15. ${ }^{[37]}$ Here, a few nanometers thick Co layer is located at different positions with respect to the surface of an optically opaque Au film. It has been demonstrated that, in this configuration, the magnetic field introduces a perturbation on the SPP wavevector due to the presence of this thin Co layer. For this particular case, $\Delta k_{s p}$ takes the form:

$$
\Delta k_{s p}(M) \approx 2 h_{1} \frac{\left(k_{0} \varepsilon_{d} \varepsilon_{A u}\right)^{2}}{\left(\varepsilon_{d}+\varepsilon_{A u}\right)\left(\varepsilon_{d}^{2}-\varepsilon_{A u}^{2}\right)} \frac{i \varepsilon_{C o}^{m o}}{\varepsilon_{C o}} \mathrm{e}^{-2 h k_{z}^{A u}},
$$

where $h_{1}$ is the Co layer thickness; $\varepsilon_{A u}$ the dielectric constant of $\mathrm{Au} ; \varepsilon_{d}$ the dielectric constant of the interface dielectric medium; $\varepsilon_{C o}$ and $\varepsilon_{C o}^{m o}$ represent the optical and MO constants of Co, respectively; $k_{z}^{A u}$ is related to the SPP field penetration in the metallic layer; and $h$ corresponds to the depth of the Co layer inside the Au film. 
This equation can also be expressed in the following way to explicitly show the dependence of the wavevector modulation with the SPP EM field intensity inside the FM layer, $\left|H_{y}^{C o}\right|^{2}:{ }^{[192]}$

$$
\Delta k_{s p} \approx h_{1} \frac{k_{0} \varepsilon_{d} \varepsilon_{A u} \sqrt{-\left(\varepsilon_{d}+\varepsilon_{A u}\right)}}{\varepsilon_{d}^{2}-\varepsilon_{A u}^{2}} \frac{i \varepsilon_{C o}^{m o}}{\varepsilon_{C o}}\left|H_{y}^{C o}\right|^{2} .
$$

As it can be seen in these equations, there are three ways of controlling the wavevector modulation: by tuning the Co layer thickness (more Co material provides more modulation); by tuning its position within the noble metal layer (being the SPP an evanescent wave, the amount of EM field inside the Co layer and therefore the modulation exponentially increase as we approach the Co layer to the surface, see Figure 15 (f)); and by modifying the dielectric material on top of the metal layer ${ }^{[193]}$ (conversely to the previous situation, in this case the change of dielectric material induces a EM field redistribution which may lead to an increased value in the Co layer, as it is shown in Figure $15(\mathrm{~g})$ ). Indeed, similarly to what was shown in the MO enhancement section regarding LSP resonances, where the FM layer could be used as a probe to determine the EM field distribution inside the metallic structure by determining the evolution of the MO activity, the analysis of the wavevector modulation can also be applied in this way.

Additionally, we can exploit the wavelength dependence of the optical and MO constants of the different materials involved and explore the spectral ranges with higher modulation. In this kind of noble metal/ferromagnetic metal multilayer systems, the wavelength dependence of the SPP wavevector modulation is dominated by the spectral dependence of the SPP electromagnetic field inside the FM layer (associated to the 
noble metal optical constants) rather than by the wavelength evolution of the MO constant. $^{[192]}$

On the other hand, the analysis of metallic thin films surrounded by two dielectric materials has been faced using ATR geometry for noble metal/ferromagnetic metal/noble metal multilayers. ${ }^{[132,78,135,136,137]}$ In these systems, comparing the angular derivative of the reflectance with the angular shape of the transversal MO signal, the value of the wavevector modulation can be determined (see Figure 16 (a)). ${ }^{[132]}$ Actually, the similitude between both curves indicates that the origin of the strong transversal MO Kerr signal under SPP excitation is due to the modification of the SPP wavevector under an applied magnetic field and the subsequent modification of the optimum angle for SPP coupling. These works have analyzed the influence that the nature of the plasmonic material, ${ }^{[135,136]}$ the amount and position of ferromagnetic material, ${ }^{[132,78]}$ and the structural quality of the multilayer interfaces ${ }^{[137]}$ have on the wavevector modulation. Roughly speaking, the effect of the amount and position of the FM layer is similar to what has been described in the single interface case. On the other hand, the role of the noble metal is directly affecting the optical constants present in the wavevector modulation expression (Eq. (9)), which have a different spectral dependence for each plasmonic material. Most importantly, it has been shown for purely plasmonic systems that the surface and interface quality of the metallic structure play a relevant role. ${ }^{[194,195,196]}$ For magnetoplasmonic multilayers, the interface sharpness between the noble metal and the ferromagnet greatly affects the optical and MO constants of the system, which in the end determine the magnetic modulation of the wavevector (see Figure $16(\mathrm{~b}))::^{[137]}$ rough noble metal-ferromagnetic metal interfaces may be visualized as effective media with reduced MO constants, whereas ultraflat interfaces make it possible to preserve the FM metal MO constants intact. 
The previously described systems only support plasmons of propagating nature. In more complex structures, such as the plasmonic crystals, modes of different character, localized and propagating, may coexist. In this case, the magnetic field dependence of the surface plasmons wavevector can be modified by the interaction between the modes. This effect has been analyzed by Torrado et $a l^{[197]}$ in a system consisting of a continuous $\mathrm{Au} / \mathrm{Co} / \mathrm{Au}$ trilayer separated of an array of $\mathrm{Au}$ nanodisks by a $\mathrm{SiO}_{2}$ spacer (see sketch in Figure 17). In this system, the SPP of the trilayer coexists with the LSP of the nanodisks, the former being excited by the grating effect of the array. The coupling between the SPP and the LSP can be controlled by changing the light angle of incidence. ${ }^{[198,199]}$ In the region of strong coupling between the localized and propagating modes, the magnetic field dependence of the wavevector of the propagating surface plasmon is reduced, due to the partial loss of propagating character of the SPP induced by the strong coupling with the LSP modes. In the same way, the LSP, typically without modulation capabilities, is now modulated by the magnetic field, again by its mixed character with the SPP counterpart (see Figure 17 (a) and (b)).

The magnetic field dependence of the SPP wavevector has also been studied in noble metal-MO dielectric systems. ${ }^{[168]}$ Analyzing the MO response of a one dimensional Au grating deposited on top of a Bi-IG film, Belotelov et al. have shown an enhancement of the TMOKE signal, which they unambiguously associate with the MO modulation of the SPP wave vector localized at the gold-garnet interface. This can be seen in Figure 18 (a) and (b), where the transmission through the structure presents different minima related with the excitation of SPPs on the system, and the MO signal shows maxima associated with these minima. They claim that this relationship between wavevector modulation and MO enhancement could be used as a tool to determine the SPP nature of the modes supported by complex structures. This is also shown in Figure 18 (a) and 
(b), where the maxima in transmission related with a Fabry-Perot resonance (hole cavity modes) do not have any associated feature in the transversal MO signal. Moreover, they show a clear example (see Figure 18 (c)) of magnetic field induced non-reciprocal effects in SPP propagation: under an applied magnetic field, SPPs of the same frequency but propagating in opposite directions have different wavevectors. This is reflected in an inversion of the peak sign associated with the SPP when reversing the angle of incidence and therefore reversing the SPP propagation direction.

Non-reciprocity in SPP propagation is indeed one of the most appealing effects that the magnetic field introduces, both from a fundamental point of view and for applications. Examples of applications based on SPP non-reciprocity will be discussed in detail in the next section. Regarding the fundamental concept, the better known example of nonreciprocity, which is indeed a consequence of the axial nature of the magnetic field, is the Faraday effect in bulk materials, where the wavevector of circularly polarized light propagating in the same direction as the applied magnetic field depends on the propagation sense. The same situation can be found in different kinds of surface waves, ${ }^{[200,201]}$ and in particular in surface plasmon polaritons. As it has been shown above, and only for the case of magnetic fields applied parallel to the interface but perpendicular to the SPP propagation, the SPP wavevector for a given frequency has a linear term that depends on the magnetic field or the magnetization: $k_{s p}=k_{s p}^{0}+\Delta k_{s p}$. Let's assume that the SPP propagates in the $x$ positive direction $\left(+k_{s p}^{0}\right)$, and we apply a magnetic field along $+y$ axis such that $\Delta k_{s p}$ is positive; then, $k_{s p}$ increases. If the SPP propagates in the negative direction, $-k_{s p}^{0}$, but the applied magnetic field is not modified, the resulting $k_{s p}$ will be reduced, as $\Delta k_{s p}$ keeps its sign (see the sketch shown in Figure 19). Therefore, for a given frequency, the wavevector of an SPP propagating 
in one way is different from that of a counter-propagating SPP under an applied magnetic field. In terms of the dispersion relation, this means that it becomes asymmetric, i.e., $\omega(\boldsymbol{k}) \neq \omega(-\boldsymbol{k})$.

This simple picture is valid for single interface, but it can be strongly modified in the case of interacting multiple interfaces, such as thin metallic films surrounded by dielectrics. For example, for a thick MO metallic film surrounded by two dielectrics, a SPP is supported in the upper interface and another one in the lower interface. For an applied magnetic field that increases the wavevector of the upper SPP, the effect on the lower SPP would be the opposite (rotate sketch 1 in Figure 19 around $y$ axis). ${ }^{[75]}$ For interacting SPPs, the wavevector modification induced by the applied magnetic field is therefore reduced. In particular, for the symmetric case of thin films embedded in a dielectric, $\Delta k_{s p}$ is zero and the dispersion relation becomes reciprocal. This problem has been treated in more detail by several authors. ${ }^{[185,202,75]}$

Non-reciprocity effects in more complex systems have also been analyzed, such as dielectric layers embedded in thin metallic films, ${ }^{[203]}$ media that combine both gyroelectric and gyromagnetic effects, ${ }^{[204]}$ interfaces composed of one gyromagnetic media and another media with negative refractive index, ${ }^{[205]}$ or plasmonic crystals exhibiting extraordinary transmission properties embedded in MO dielectric materials. ${ }^{[206]}$ Finally, other non-reciprocity effects have also been observed in the scattering of SPPs by magnetic nanoparticles. ${ }^{[207]}$

\section{Applications}

As mentioned in the introduction, the fields of applications of magnetoplasmonic structures lie in the domains of sensing and telecommunication devices. Here we will account for the main demonstrations achieved so far in both fields combining magnetic and plasmonic functionalities in an intertwined fashion. 


\subsection{Sensing}

Surface plasmon resonance (SPR) platforms are currently of common use in gas and biosensing applications. They are basically refractive index change sensors that rely on the sensitivity of the SPP wavevector to changes in the dielectric environment, thanks to the evanescent nature of this mode. ${ }^{[46]}$ For this application, a Au layer is attached to a prism to conform an ATR configuration and the reflectivity curve (or the intensity at a specific point of this curve) is recorded as a function of the dielectric environment changes. Recently, magnetoplasmonic systems have been proposed as alternative transducers to the Au layers in these well established SPR sensors. These systems, denoted magneto-optical SPR (MOSPR) sensors, employ the transversal MO Kerr signal as the sensing magnitude instead of the reflectivity intensity (see Figure 20 (a) and (b)). This approach was first proposed by Sepúlveda et al. ${ }^{[36]}$ using Co/Au layers and finding a threefold enhancement in the limit of detection with respect to standard SPR sensing platforms (Figure 20 (c)). Later, similar structures, both with $\mathrm{Au} / \mathrm{Co} / \mathrm{Au}$ and $\mathrm{Au} / \mathrm{Fe} / \mathrm{Au}$ trilayers, have been used to show their suitability in biosensing applications. ${ }^{[73,74,208,209]}$ In these works, a careful analysis of the optimum multilayer structure to maximize the system sensitivity is performed, finding that a trade-off in MO response and optical absorption (this last determines the width of the plasmon resonance) is fundamental to achieve the optimum result. An alternative functionalization of the surface of the transducer, for example with highly porous, columnar $\mathrm{TiO}_{2}$ also opens new routes in gas sensing. ${ }^{[72]}$

Although not as extended as the ones based on propagating plasmons, LSPs based sensors are receiving an increasing attention. Very recently, Bonanni et al. ${ }^{[89]}$ proposed the use of Ni nanodisks as good candidates for the development of biosensing devices. This work points out the advantages of this system where the inverse of the MO rotation 
would be taken as the detection signal: the sensitivity to refractive index changes is similar to that of the absorption peak position in Au nanoparticles, but with an improved figure of merit (shift divided by FWHM of resonance).

\subsection{Nanophotonic devices: Integrated optical isolators and modulators}

As mentioned before, under a magnetic field in transversal configuration, SPP propagation is non-reciprocal, i.e., the wavevector propagating in one direction is different from that propagating in the other $(\omega(\boldsymbol{k}) \neq \omega(-\boldsymbol{k}))$. Non-reciprocity of SPPs offers an interesting way to design integrated optical isolators, elements that are likely to be highly demanded in the context of nanophotonic circuitry, and some proposals, most of them theoretical but also some experimental demonstrations, have been put forward.

Starting with the theoretical schemes, Sepúlveda et al. ${ }^{[75]}$ made a general study of the effect of the magnetic field in the long-range SPP of a thin Au layer surrounded by garnet films (or only one garnet film and one passive dielectric) and discussed the applicability of this system to develop an optical isolator in a Mach-Zehnder interferometer configuration. Similarly, Khurgin ${ }^{[210]}$ offers an analysis of the propagation of SPPs in a noble metal (Ag)/garnet interface and in a $\mathrm{Ag}$ thin film surrounded by passive dielectric on one side and garnet on the other, and he proposes the development of an optical isolator in a Mach-Zehnder configuration with one active arm and one passive one. Yu et al. ${ }^{[62]}$ suggest a different approach by coupling a $\mathrm{Au}$ film that sustains SPPs with a semi-infinite photonic crystal in such a way than when a magnetic field is applied the "forward"-SPP can propagate but the "backwards"-SPP cannot propagate because it is in the gap of the photonic crystal. Although the idea behind this proposal is quite attractive, a practical realization dealing with pure Au films does not seem feasible because of the tiny $\mathrm{MO}$ response of $\mathrm{Au}$, as already discussed in 
this review. The introduction of MO active photonic crystals ${ }^{[211]}$ or magnetoplasmonic multilayers in this kind of system could provide enough magnetic-field wavevector modulation to make the effect realistic. Finally, Montoya et al. ${ }^{[212]}$ propose the coupling of a dielectric waveguide mode ( $\mathrm{InP} / \mathrm{InGaAsP} / \mathrm{InP})$ with a SPP sustained in a pure ferromagnetic metal (Fe)/air interface. When a magnetic field is applied, coupling exists for the backward-propagating mode but not for the forward-propagating one. As a consequence, only the forward propagating wave is efficiently transmitted through the waveguide, as the backwards one is finally absorbed in the ferromagnetic metal. An important advantage of this scheme is that it contains no garnets, which so far pose important problems in terms of fabrication and integration, as garnet deposition is not easily compatible with the fabrication techniques usual in integrated silicon photonics.

From the experimental point of view, there is one demonstration carried out by Van Parys et al. ${ }^{[213,214]}$ and also based on the coupling of a dielectric waveguide (AlGaInAs/InP in this case, with amplification included) with an SPP supported by a $\mathrm{Co}_{50} \mathrm{Fe}_{50}$ alloy cladding located on top of the waveguide (see Figure 21 (a)). They demonstrate a TM-mode AlGaInAs/InP amplifying optical waveguide isolator with an experimental isolation ratio of $99 \mathrm{~dB} / \mathrm{cm}$. An analogous proposal but for TE-mode waveguides can be found in the work of Shimizu et al. ${ }^{[215,216]}$ They used here InGaAsP/InP semiconductor optical amplifier waveguides combined with a Fe layer to provide the non-reciprocity. In this case, in order to keep the appropriate geometry, the ferromagnetic layer is placed on one sidewall of the waveguide, and the magnetic field is applied perpendicular to the waveguide (see Figure 21 (b)). A performance of 14 $\mathrm{dB} / \mathrm{mm}$ was demonstrated in this case.

Finally, the modification of the SPP wavevector by an external applied magnetic field also allows the development of integrated optical modulators. The first proposal, ${ }^{[217]}$ 
demonstrated only on a theoretical basis, uses SPPs excited in a Ag/bismuth-substituted yttrium iron garnet interface. The modulator geometry is based on the ATR configuration, being therefore relatively bulky and not fully integrable, and its basis of operation relies on the modification of the SPP coupling angle under an applied magnetic field perpendicular to the plane of incidence due to the SPP wavevector modification. The analysis of this device shows that it is able to provide high efficiency modulation, around 50\%, at high speed, i.e. GHz regime, in the IR range (at $1550 \mathrm{~nm}$ ). Some years later a more compact geometry, based on a plasmonic interferometer engraved in a noble metal/ferromagnetic metal multilayer, was experimentally developed (see Figure 15). ${ }^{[37,192,193]}$ The magnetoplasmonic interferometer consists of a slit-groove pair, with the groove slightly tilted with respect to the slit, milled in a $\mathrm{Au} / \mathrm{Co} / \mathrm{Au}$ trilayer (see the sketch in Figure 15 (a)). For an incident beam illuminating the interferometer, a portion of the light hits the groove and it is able to excite a SPP at the metal-air interface which propagates towards the slit $\left(I_{s p}\right)$ and another portion travels through the slit $\left(I_{r}\right)$. When the surface plasmon reaches the slit, it is again reconverted into radiative light and traverses the slit. The light intensity at the other side of the slit is therefore given by the interference of these two contributions:

$$
I=I_{r}^{2}+I_{s p}^{2}+2 \sqrt{I_{r} I_{s p}} \cos \left(k_{s p} d(x)+\varphi_{0}\right)
$$

with $d$ the distance between the slit and the groove and $\varphi_{0}$ and arbitrary phase introduced at the SPP excitation and decoupling. As $d$ varies with the position along the slit due to the angle between groove and slit, the cosine argument changes and a full interference pattern is produced (see Figure 15 (b)). When a magnetic field is applied parallel to the slit (transversal configuration), the SPP wavevector is modified and as a 
consequence the interference pattern is shifted. This results in a modulation of the intensity transmitted through the slit at each point, whose amplitude under direction reversal of an applied magnetic field high enough to saturate the sample $(\approx 20 \mathrm{mT})$ is given by:

$$
I_{m p}=-4 \sqrt{I_{r} I_{s p}} \Delta k_{s p} d \sin \left(k_{s p}^{0} d+\varphi_{0}\right) .
$$

As it can be seen from Eq. (12), the modulation amplitude is proportional to the product of the SPP wavevector modification and the slit-groove distance, $\Delta k_{s p} \times d$. In the case of $\mathrm{Au} / \mathrm{Co} / \mathrm{Au}$ layers, taking slit-groove distances of $20 \mu \mathrm{m}$ and working at $800 \mathrm{~nm}$, intensity modulations of around $2 \%$ were demonstrated. However, this can be increased by adding a dielectric layer on top of the interferometers, which as it has been previously mentioned increases $\Delta k_{s p},{ }^{[193]}$ by optimizing the properties of the MO layer, for example using the above described Fe layers with flat interfaces; ${ }^{[137]}$ and by working in the spectral range that maximizes the figure of merit $\Delta k_{s p} \times L_{s p}$, being $L_{s p}$ the SPP propagation distance. ${ }^{[192]}$ In fact, for a magnetoplasmonic interferometer of $\mathrm{Au} / \mathrm{Fe} / \mathrm{Au}$ covered with a dielectric of refractive index $n=2$, working in the spectral range around $950 \mathrm{~nm}$ and with the slit placed at a distance of $3 L_{s p}$ from the groove, which still provides defined interferences, modulations around $12 \%$ can be achieved, which is close to the $15-20 \%$ values reported in the literature for other integrated plasmonic modulators based on non magneto-optical effects. ${ }^{[48]}$ Moreover, the magneto-optical effect has an advantage over other driving agents proposed in the design of integrated photonic modulators, such as thermo-optical or electro-optical effects: switching can be much faster. The generation of magnetic fields by integrated electronic circuits can 
easily reach speeds in the $\mathrm{GHz}$ regime, and modulations in the $\mathrm{THz}$ regime could also be achieved by controlling the magnetization with femtosecond light pulses. ${ }^{[56]}$

\section{Other phenomena associated to systems with plasmonic and ferromagnetic functionalities}

As mentioned in the introduction, this review focus on the rich phenomenology resulting in systems with intertwined magnetic and plasmonic properties. These structures are formed mainly combining plasmonic materials and magnetic ones, in the so-called magnetoplasmonic structures. Additionally, there are in the literature a relevant number of studies devoted to structures that also combine both type of materials, but where the plasmon effects and the magnetic ones are treated in an uncoupled way. This kind of structures can be defined as magnetic-plasmonic systems, and in the last years have appeared several relevant applications associated with them. For the sake of completeness, we present in this last section of the review a few examples of these magnetic-plasmonic systems, and refer the reader to the specialized literature to obtain more information on this approach.

One of the most interesting examples of non-interacting but coexistent magnetic and plasmonic properties can be found in particles containing magnetic elements (typically an iron oxide) and noble metals (usually gold). The reason for the intense activity and interest in these of systems lies in the possibility of application for detection and treatment (theranostics) of malign cells. The range of application is large, e.g. various types of cancer, Alzheimer, virus-induced infections, etc. ${ }^{[218,219]}$ That wide range of applications comes from the different capabilities endorsed by each element. Plasmonic nanoparticles can be used for imaging, since their tunable properties via size and shape permit to place the resonance at the desirable wavelength according the tissue to be imaged. ${ }^{[220,221,222]}$ On the other hand, the magnetic counterpart has been used as contrast 
agent in magnetic resonance imaging, and it is essential for magnetic separation. ${ }^{[223,224,225]}$ As mentioned, magnetic-plasmonic systems are not only useful for imaging but also for therapy. In that case, both are used for hyperthermia: in the case of the noble metal the plasmon resonance is the mean to increase temperature, while high magnetic fields are needed for the magnetic case. Their combination permits stabilizing magnetic nanoparticles and easy biofunctionalization using thiol-based protocols. ${ }^{[226]}$

These kind of systems also find an ample spectrum of applications in biosensing areas, like the novel approach proposed by Scrittwieser et al. for homogeneous real-time immunodiagnostics, based on the plasmon related optical detection of the relaxation dynamics of magnetic nanoparticles. ${ }^{[227]}$ If the nanomaterial also presents shape anisotropy, then polarization might play an important role, improving the performance as bio-probes. ${ }^{[28,229]}$ Another potential application of anisotropic magnetic-plasmonic systems is in the field of magnetic tweezers and magnetic separation. ${ }^{[228]}$

A different aspect that has been studied is the effect of plasmon excitation in magnetic properties different from the MO activity. In this sense, C. de Julián and co-workers have studied the coupling between the magnetic and optical properties of $\mathrm{Au}-\mathrm{Fe}$ nanoparticles, exploring their plasmonic and MO properties, as well as the photoinduced effects on their coercivity. ${ }^{[230,231]}$

\section{Conclusions and perspectives}

Nowadays the tools that nanotechnology offers allow us to devise novel structures where EM resonances and the subsequent subwavelength field distribution may be tailored at will. This is the most relevant driving force in plasmonics, which, in addition to the versatility that combining constituting elements with different functionalities (magnetic and plasmonic in this case) offers, brings as a consequence the possibility to 
design quite a large variety of systems where plasmonic and magnetic properties are intertwined, one acting on each other.

From a historical perspective, the paradigmatic example is the plasmon resonance enhanced MO activity in a wide variety of systems, with a wide range of structural complexity covering from isolated nanoparticles (including dimers, core-shell, nanodisks with multilayered internal architecture, etc...) to continuous thin films with more or less complex internal multilayered structure, including the combination of both. The enhancement in the MO activity observed in systems upon plasmon excitation can be up to several orders of magnitude, making magnetoplasmonic structures interesting candidates for a number of applications. Of them, sensing (MO-SPR sensing) is the one currently more developed and settled.

Regarding the physical mechanisms responsible of this enhancement, both the optical and MO parts of the total MO response of a system play a relevant role, being in some cases the MO contribution more important, while the optical one dominates in others. For a fundamental understanding of this phenomenology and to favour the design of future systems with optimized MO activity, it is important to keep in mind that, for systems where the pure MO contribution dominates, the EM field intensity at the MO active layer will be in the end the key factor to determine the global MO activity of the whole system. For this reason, the EM field engineering in subwavelength scales provided by the plasmonic structures make them perfectly suited for this purpose. The final goal would therefore be to fabricate a system whose plasmonic resonances would concentrate most of the EM field in the MO active component, minimizing it in all the rest non MO, but somehow lossy ones. This way, those novel systems could become an alternative to state of the art dielectric MO materials, like garnets. Another possible path for MO enhancement is the use of systems with propagating surface plasmons in 
transversal configuration, where the magnetic field induced SPP wavevector modulation also translates in an increase of the system MO activity.

On the reverse direction, it is also relevant the potentiality of generating active plasmonic systems with ultrafast response times exploiting the plasmon wavevector modulation under the application of a magnetic field in magnetoplasmonic systems. One envisaged application in this case would be ultrafast magnetoplasmonic modulators in the visible. Moreover, the non-reciprocal property exhibited by these systems can also be exploited for the design of optical isolators for integrated photonic circuits, components that are highly demanded to prevent circuit damages.

Additionally to the fundamental understanding and development of all these properties, an important angle of the magnetoplasmonic field deals with the materials point of view. One needs to find novel materials which simultaneously exhibit good plasmonic and MO properties, with low losses and realistically transferable to the production area. If these materials do not exist in nature, nanotechnology allows us to combine different materials that, in a partial but complementary way, fulfill the desired requirements, engineering the plasmonic and MO characteristics and giving rise to advanced systems which finally present the optimized magnetoplasmonic properties. For this purpose, in many cases it will be necessary to combine noble metals, ferromagnetic metals, ferromagnetic and non ferromagnetic dielectrics in a smart way. All the materials science investigation needed for this development is also extremely relevant.

Currently, there is an increasing number of research groups in the world working in this area, and the faced problems evolve in a dynamic way adapting to the scientific and societal needs. For example, in the future systems with large MO and low absorption will be highly desirable, as they would allow the design of MO-based spatial light modulators that will afford the transmission of holograms at high speed for a true 3D 
vision. Regarding the expanding field of sensing and biosensing, MO sensing not only with SPP configurations but also with LSP ones is foreseen, with expected even higher sensitivities. The field of integrated non-reciprocal devices is really open, as the choice of systems offering non-reciprocity is limited, and there are many alternatives that may be considered here.

Finally, graphene is nowadays the most studied novel material due to the wide variety of exciting properties that it exhibits. In addition to the purely plasmonic properties that make graphene an interesting research topic in this area, ${ }^{[232,233]}$ other aspects closely related to the present review such as its MO activity have also been recently put forward, finding for example Faraday rotations of several degrees for magnetic fields of a few Teslas in single- and multilayer graphene structures. ${ }^{[234]}$ More interestingly, the potential that this MO activity of graphene offers in terms of applications in the far IR and terahertz regime has recently been boosted by the experimental finding of the graphene plasmons effects in the MO activity. ${ }^{[235,236]}$ This implies that a great deal of interest regarding the applications of magnetoplasmons in graphene for fast tunable ultrathin infrared and terahertz MO devices is clearly foreseen. As a matter of fact, the interest in the terahertz regime is increasing with the appearance of new EM sources and detectors that have opened a new range of applications, ${ }^{[237]}$ motivating new studies of plasmonic devices in this frequency range. ${ }^{[238,239,240]}$ This offers good opportunities also for magnetoplasmonic devices using n-doped semiconductors. ${ }^{[241,242]}$

\section{Acknowledgements}

We acknowledge Ll. Balcells for supplying us with the hysteresis loops shown in Figure 3 and funding support from the Spanish MICINN ("FUNCOAT" CONSOLIDER INGENIO 2010 CSD2008-00023, MAPS MAT2011-29194-C02-01, and PLASMAR 
MAT2010-10123-E), and the Comunidad de Madrid ("NANOBIOMAGNET", S2009/MAT-1726 and “MICROSERES-CM”, S2009/TIC-1476).

\section{References:}

[1] K. W. Chiu, J. J. Quinn, Phys. Rev. B 1972, 5, 4707.

[2] J. J. Brion, R. F. Wallis, A. Hartstein, E. Burstein, Phys. Rev. Lett. 1972, 28, 1455.

[3] K. W. Chiu, J. J. Quinn, Phys. Rev. Lett. 1972, 29, 600.

[4] E. D. Palik, R. Kaplan, R. W. Gammon, H. Kaplan, J. J. Quinn, R. F. Wallis, Phys. Lett. A 1973, 45, 143.

[5] A. Hartstein, E. Burstein, Solid State Commun. 1974, 14, 1223.

[6] A. Hartstein, E. Burstein, E. D. Palik, R. W. Gammon, B. W. Henvis, Phys. Rev. B $\mathbf{1 9 7 5}, 12,3186$.

[7] W. Reim, O. E. Hüsser, J. Schoenes, E. Kaldis, P. Wachter, K. Seiler, J. Appl. Phys. 1984, 55, 2155.

[8] H. Feil, C. Haas, Phys. Rev. Lett. 1987, 58, 65.

[9] J. Schoenes, W. Reim, Phys. Rev. Lett. 1988, 60, 1988.

[10] H. Feil, C. Haas, Phys. Rev. Lett. 1988, 60, 1989.

[11] T. Katayama, Y. Suzuki, H. Awano, Y. Nishihara, N. Koshizuka, Phys. Rev. Lett. 1988, 60, 1426.

[12] W. Reim, D. Weller, Appl. Phys. Lett. 1988, 53, 2453.

[13] E. Huber, E. E. Marinero, Appl. Phys. A 1988, 47, 131.

[14] W. A. McGahan, L.-Y. Chen, Z. S. Shan, D. J. Sellmyer, J. A. Woollan, Appl. Phys. Lett. 1989, 55, 2479.

[15] W. Reim, D. Weller, IEEE Trans. Magn. 1989, 25, 3752.

[16] R. Nies, F. R. Kessler, Phys. Rev. Lett. 1990, 64, 105. 
[17] V. I. Safarov, V. A. Kosobukin, C. Hermann, G. Lampel, J. Peretti, C. Marlière, Phys. Rev. Lett. 1994, 73, 3584.

[18] C. Hermann, V. A. Kosobukin, G. Lampel, J. Peretti, V. I. Safarov, P. Bertrand, Phys. Rev. B 2001, 64, 235422.

[19] S.-Y. Wang, W.-M. Zheng, D.-L. Qian, R.-J. Zhang, Y.-X. Zheng, S.-M. Zhou, Y.-M. Yang, B.-Y. Li, L.-Y. Chen, J. Appl. Phys. 1999, 85, 5121.

[20] W.-M. Zheng, L.-Y. Chen, J.-H. Chu, Phys. Status Solidi B 1999, 214, 463.

[21] W.-M. Zheng, S.-Y. Wang, D.-L. Qian, Y.-X. Zheng, Y.-M. Yang, B.-Y. Li, L.-Y. Chen, J. Magn. Magn. Mater. 1999, 198-199, 210.

[22] E. Popov, M. Neviere, J. Opt. Soc. Am. A 2001, 18, 2886.

[23] L. Li, J. Opt. A: Pure Appl. Opt. 2003, 5, 345.

[24] A. Garcia-Martin, G. Armelles, S. Pereira, Phys. Rev. B 2005, 71, 205116.

[25] D. A. Smith, K. L. Stokes, Opt. Express 2006, 14, 5746.

[26] A. B. Khanikaev, A. V. Baryshev, A. A. Fedyanin, A. B. Granovsky, M. Inoue, Opt. Expr. 2007, 15, 6612.

[27] V. I. Belotelov, D. A. Bykov, L. L. Doskolovich, A. N.Kalish, A. K. Zvezdin, J. Opt. Soc. Am. B 2009, 26, 1594.

[28] S. Albaladejo, R. Gómez-Medina, L. S. Froufe-Pérez, H. Marinchio, R. Carminati, J. F. Torrado, G. Armelles, A. García-Martín, J. J. Sáenz, Opt. Express 2010, 18, 3556.

[29] R. Alcaraz de la Osa, J. M. Saiz, F. Moreno, P. Vavassori, A. Berger, Phys. Rev. B 2012, 85, 064414.

[30] B. Caballero, A. García-Martín, J. C. Cuevas, Phys. Rev. B 2012, 85, 245103.

[31] S. A. Maier, Plasmonics: fundamentals and aplications, Springer, 2007.

[32] W. L. Barnes, A. Dereux, T. W. Ebbesen, Nature 2003, 424, 824. 
[33] I. L. Lyubchanskii, N. N. Dadoenkova, M. I. Lyubchanskii, E. A. Shapovalov, T. Rasing, J. Phys. D: Appl. Phys. 2003, 36, R277.

[34] M. Inoue, R. Fujikawa, A. Baryshev, A. Khanikaev, P. B. Lim, H. Uchida, O. Aktsipetrov, A. Fedyanin, T. Murzina, A. Granovsky, J. Phys. D: Appl. Phys. 2006, 39, R151.

[35] M. Levy, A. A. Jalali, X. Huang, J. Mater. Sci.: Mater. Electron. 2009, 20, S43.

[36] B. Sepúlveda, A. Calle, L. M. Lechuga, G. Armelles, Opt. Lett. 2006, 31, 1085.

[37] V.V. Temnov, G. Armelles, U. Woggon, D. Guzatov, A. Cebollada, A. GarcíaMartín, J.M. García-Martín, T. Thomay, A. Leitenstorfer, R. Bratschitsch, Nat. Photonics 2010, 4, 107.

[38] C. F. Bohren, D. R. Huffman, Absorption and Scattering of Light by Small Particles, Wiley-VCH, Weinheim, Germany, 2004.

[39] E. Hutter, J. H. Fendler, Adv. Mater. 2004, 16, 1685.

[40] H. Raether, Surface Plasmons on Smooth and Rough Surfaces and on Gratings, Springer-Verlag, Berlin, 1988.

[41] A. V. Zayats, I. I. Smolyaninov, J. Opt. A: Pure Appl. Opt. 2003, 5, S16.

[42] E. Ozbay, Science 2006, 311, 189.

[43] D. K. Gramotnev, S. I. Bozhevolnyi, Nat. Photonics 2010, 4, 83.

[44] M. I. Stockman, Phys. Today 2011, 64, 39.

[45] J. N. Anker, W. P. Hall, O. Lyandres, N. C. Shah, J. Zhao, R. P. V. Duyne, Nat. Mater. 2008, 7, 442.

[46] J. Homola, Chem. Rev. 2008, 108, 462.

[47] T. Nikolajsen, K. Leosson, S. I. Bozhevolnyi, Appl. Phys. Lett. 2004, 85, 5833.

[48] J. Gosciniak, S. I. Bozhevolnyi, T. B. Andersen, V. S. Volkov, J. KjelstrupHansen, L. Markey, A. Dereux, Opt. Express 2010, 18, 1207. 
[49] M. J. Dicken, L. A. Sweatlock, D. Pacifici, H. J. Lezec, K. Bhattacharya, H. A. Atwater, Nano Lett. 2008, 8, 4048.

[50] J. A. Dionne, K. Diest, L. A. Sweatlock, H. A. Atwater, Nano Lett. 2009, 9, 897.

[51] A. Agrawal, C. Susut, G. Stafford, U. Bertocci, B. McMorran, H. J. Lezec, A. A. Talin, Nano Lett. 2011, 11, 2774.

[52] D. Pacifici, H. J. Lezec, H. A. Atwater, Nat. Photonics 2007, 1, 402.

[53] R. A. Pala, K. T. Shimizu, N. A. Melosh, M. L. Brongersma, Nano Lett. 2008, 8, 1506.

[54] K. F. MacDonald, Z. L. Sámson, M. I. Stockman, N. I. Zheludev, Nat. Photonics 2009, 3, 55.

[55] V. V. Temnov, K. Nelson, G. Armelles, A. Cebollada, T. Thomay, A. Leitenstorfer, R. Bratschitsch, Opt. Express, 2009, 17, 8423.

[56] A. V. Kimel, A. Kirilyuk, P. A. Usachev, R. V. Pisarev, A. M. Balbashov, Th Rasing, Nature 2005, 435, 655.

[57] M. Faraday, Phil. Trans. R. Soc. Lond. 1846, 136, 1.

[58] J. Kerr, Phil. Mag. 1877, 3, 321.

[59] J. Kerr, Phil. Mag. 1878, 5, 161.

[60] A. K. Zvezdin, V. A. Kotov, Modern Magnetooptics and Magnetooptical Materials, IOP Publishing Ltd, Bristol, UK, 1997.

[61] T. K. Xia, P. M. Hui, D. Stroud, J. Appl. Phys. 1990, 67, 2736.

[62] Z. Yu, G. Veronis, Z. Wang, S. Fan, Phys. Rev. Lett. 2008, 100, 023902.

[63] Q. Majorana, Nuovo Cimento 1944, 2, 1.

[64] E. A. Stern, J. C. McGroddy, W. E. Garte, Phys. Rev. 1964, 135, A1306.

[65] S. E. Schnatterly, Phys. Rev. 1969, 183, 664.

[66] P. Haefner, E. Luck, E. Mohler, Phys. Status Solidi B 1994, 185, 289. 
[67] H. Ebert, Rep. Prog. Phys. 1996, 59, 1665.

[68] P. Oppeneer, V. Antonov, in Spin-Orbit-Influenced Spectroscopies of Magnetic Solids (Eds.: H. Ebert, G. Schütz), Springer, Berlin/Heidelberg, Germany 1996, p. 29.

[69] P. B. Johnson, R. W. Christy, Phys. Rev. B 1972, 6, 4370.

[70] P. B. Johnson, R. W. Christy, Phys. Rev. B 1974, 9, 5056.

[71] G. Armelles, A. Cebollada, A. García-Martín, J. M. García-Martín, M. U. González, J. B. González-Díaz, E. Ferreiro-Vila, J. F. Torrado, J. Opt. A: Pure Appl. Opt. 2009, 11, 114023.

[72] M. G. Manera, G. Montagna, E. Ferreiro-Vila, L. González-García, J. R. SánchezValencia, A. R. González-Elipe, A. Cebollada, J. M. García-Martín, G. Armelles, R. Rella, J. Mater. Chem. 2011, 21, 16049.

[73] D. Regatos, D. Fariña, A. Calle, A. Cebollada, B. Sepúlveda, G. Armelles L. M. Lechuga, J. Appl. Phys, 2010, 108, 054502.

[74] D. Regatos, B. Sepúlveda, D. Fariña, L. G. Carrascosa L. M. Lechuga. Opt. Express 2011, 19, 8336.

[75] B. Sepúlveda, L. M. Lechuga, G. Armelles, J. Lightwave Technol. 2006, 24, 945.

[76] P. Bertrand, C. Hermann, G. Lampel, J. Peretti, V. I. Safarov, Phys. Rev. B 2001, $64,235421$.

[77] N. Bonod, R. Reinisch, E. Popov, M. Nevière, J. Opt. Soc. Am. B 2004, 21, 791.

[78] C. Clavero, K. Yang, J. R. Skuza, R. A. Lukaszew, Opt. Express 2010, 18, 7743.

[79] D. Meneses-Rodríguez, E. Ferreiro-Vila, P. Prieto, J. Anguita, M. U. González, J. M. García-Martín, A. Cebollada, A. García-Martín, G. Armelles, Small 2011, 7, 3317. [80] P. M. Hui, D. Stroud, Appl. Phys. Lett. 1987, 50, 950.

[81] N. A. Yusuf, A. A. Rousan, H. M. El-Ghanem, J. Appl. Phys. 1988, 64, 2781.

[82] V. G. Kravets, A. K. Petford-Long, A. F. Kravetz, Physica E 1999, 4, 292. 
[83] V. G. Kravets, A. K. Petford-Long, A. F. Kravets, J. Appl. Phys. 2000, 87, 1762.

[84] V. G. Kravets, L. V. Poperenko, Opt. Spectrosc. 2008, 104, 610.

[85] S. Melle, J. L. Menéndez, G. Armelles, D. Navas, M. Vázquez, K. Nielsch, R. B. Wehrspohn, U. Gösele, Appl. Phys. Lett. 2003, 83, 4547.

[86] J. B. González-Díaz, A. García-Martín, G. Armelles, D. Navas, M. Vázquez, K. Nielsch, R. B. Wehrspohn, U. Gösele, Adv. Mater. 2007, 19, 2643.

[87] J. B. González-Díaz, A. García-Martín, G. Armelles, Nanoscale Res. Lett. 2011, 6, 408.

[88] J. Chen, P. Albella, Z. Pirzadeh, P. Alonso-González, F. Huth, S. Bonetti, V. Bonanni, J. Åkerman, J. Nogués, P. Vavassori, A. Dmitriev, J. Aizpurua, R. Hillenbrand, Small 2011, 7, 2341.

[89] V. Bonanni, S. Bonetti, T. Pakizeh, Z. Pirzadeh, J. Chen, J. Nogues, P. Vavassori, R. Hillenbrand, J. Akerman, A. Dmitriev, Nano Lett. 2011, 11, 5333.

[90] B. Sepúlveda, J. B. González-Díaz, A. García-Martín, L. M. Lechuga, G. Armelles, Phys. Rev. Lett. 2010, 104, 147401.

[91] A.V. Chetvertukhin, A.A. Grunin, A.V. Baryshev, T.V. Dolgova, H. Uchida, M. Inoue, A.A. Fedyanin, J. Magn. Magn. Mater. 2012, 324, 3516.

[92] Y. Li, Q. Zhang, A. V. Nurmikko, S. Sun, Nano Lett. 2005, 5, 1689.

[93] J. B. González-Díaz, A. García-Martín, J. M. García-Martín, A. Cebollada, G. Armelles, B. Sepúlveda, Y. Alaverdyan, M. Käll, Small 2008, 4, 202.

[94] F. E. Moolekamp, K. L. Stokes, IEEE Trans. Magn. 2009, 45, 4888.

[95] P. K. Jain, Y. Xiao, R. Walsworth, A. E. Cohen, Nano Lett. 2009, 9, 1644.

[96] R. K. Dani, H. Wang, S. H. Bossmann, G. Wysin, V. Chikan, J. Chem. Phys. 2011, 135, 224502. 
[97] G. Armelles, A. Cebollada, A. García-Martín, J. M. Montero-Moreno, M. Waleczek, K. Nielsch, Langmuir 2012, 28, 9127.

[98] L. Wang, K. Yang, C. Clavero, A. J. Nelson, K. J. Carroll, E. E. Carpenter, R. A. Lukaszew, J. Appl. Phys. 2010, 107, 09B303.

[99] L. Wang, C. Clavero, Z. Huba, K. J. Carroll, E. E. Carpenter, D. Gu, R. A. Lukaszew, Nano Lett. 2011, 11, 1237.

[100] G. X. Du, T. Mori, M. Suzuki, S. Saito, H. Fukuda, M. Takahashi, Appl. Phys. Lett. 2010, 96, 081915.

[101] G. X. Du, T. Mori, M. Suzuki, S. Saito, H. Fukuda, M. Takahashi, J. Appl. Phys. 2010, 107, 09A928.

[102] G. X. Du, T. Mori, S. Saito, M. Takahashi, Phys. Rev. B 2010, 82, 161403.

[103] Y. Demidenko, D. Makarov, V. Lozovski, J. Opt. Soc. Am. B 2010, 27, 2700.

[104] G. Armelles, J. B. González-Díaz, A. García-Martín, J. M. García-Martín, A. Cebollada, M. U. González, S. Acimovic, J. Cesario, R. Quidant, G. Badenes, Opt. Express 2008, 16, 16104.

[105] G. X. Du, S. Saito, M. Takahashi, Appl. Phys. Lett. 2011, 99, 191107.

[106] G. X. Du, S. Saito, M. Takahashi, IEEE Trans. Magn. 2011, 47, 3167.

[107] J. B. González-Díaz, B. Sepúlveda, A. García-Martín, G. Armelles, Appl. Phys. Lett. 2010, 97, 043114.

[108] T. Pakizeh, M. S. Abrishamian, N. Granpayeh, A. Dmitriev, M. Käll, Opt. Express 2006, 14, 8240.

[109] A. Dmitriev, T. Pakizeh, M. Käll, D. S. Sutherland, Small 2007, 3, 294.

[110] T. Pakizeh, A. Dmitriev, M. S. Abrishamian, N. Granpayeh, M. Käll, J. Opt. Soc. Am. 2008, 25, 659 .

[111] E. Prodan, C. Radloff, N. J. Halas, P. Nordlander, Science 2003, 302, 419. 
[112] M. Abe, J. Kuroda, J. Appl. Phys. 2002, 91, 7305.

[113] M. Abe, T. Suwa, Phys. Rev. B 2004, 70, 235103.

[114] M. Abe, S. Takeshi, J. Appl. Phys. 2005, 97, 10M514.

[115] J. C. Banthí, D. Meneses-Rodríguez, F. García, M.U. González, A. GarcíaMartín, G. Armelles, Adv. Mater. 2012, 24, OP36.

[116] J. C. Banthí, D. Meneses-Rodríguez, F. García, M. U. González, A. GarcíaMartín, A. Cebollada, G. Armelles, F. J. Palomares, Proc. of SPIE 2012, 8424, 84241X.

[117] H. Fredriksson, Y. Alaverdyan, A. Dmitriev, C. Langhammer, D. S. Sutherland, M. Zäch, B. Kasemo, Adv. Mater. 2007, 19, 4297.

[118] S. Tomita, T. Kato, S. Tsunashima, S. Iwata, M. Fuji, S. Hayashi, Phys. Rev. Lett. 2006, 96, 167402 .

[119] R. Fujikawa, A.V. Baryshev, J. Kim, H. Uchida, M. Inoue, J. Appl. Phys. 2008, 103, $07 \mathrm{D} 301$.

[120] H. Uchida, Y. Masuda, R. Fujikawa, A. V. Baryshev, M. Inoue, J. Magn. Magn. Mater. 2009, 321, 843.

[121] H. Uchida, Y. Mizutani, Y. Nakai, A. A. Fedyanin, M. Inoue, J. Phys. D: Appl. Phys. 2011, 44, 064014.

[122] S. M. Hamidi, M. M. Tehranchi, S. Sadeghi, J. Phys. D: Appl. Phys. 2011, 44, 305003.

[123] I. D. Mayergoyz, G. Lang, L. Hung, S. Tkachuk, C. Krafft, O. Rabin, J. Appl. Phys. 2010, 107, 09A925.

[124] S. Tkachuk, G. Lang, C. Krafft, O. Rabin, I. Mayergoyz, J. Appl. Phys. 2011, 109, $07 \mathrm{~B} 717$.

[125] P. E. Ferguson, O. M. Stafsudd, R. F. Wallis, Physica B+C 1977, 89, 91.

[126] R. D. Olney, R. J. Romagnoli, P. E. Ferguson, J. Opt. Soc. Am B 1986, 3, 1526. 
[127] T.W. Ebessen, H.J.Lezec, H.F. Ghaemi, T. Thio, P.A. Wolf, Nature 1998, 391, 667.

[128] V. A. Kosobukin, Solid State Commun. 1997, 101, 497.

[129] N. Richard, A. Dereux, T. David, E. Bourillot, J. P. Goudonnet, F. Scheurer, E. Beaurepaire, G. Garreau, Phys. Rev. B 1999, 59, 5936.

[130] N. Richard, A. Dereux, E. Bourillot, T. David, J. P. Goudonnet, F. Scheurer, E. Beaurepaire, Phys. Status Solidi A 1999, 175, 225.

[131] L. Sapienza, D. Zerulla, Phys. Rev. B 2009, 79, 033407.

[132] J. B. González-Díaz, A. García-Martín, G. Armelles, J. M. García-Martín, C. Clavero, A. Cebollada, R. A. Lukaszew, J. R. Skuza, D. P. Kumah, R. Clarke, Phys. Rev. B 2007, 76, 153402.

[133] Y. Demidenko, D. Makarov, O. G. Schmidt, V. Lozovski, J. Opt. Soc. Am. B 2011, 28, 2115.

[134] L. G. C. Melo, A. D. Santos, L. M. Alvarez-Prado, Y. Souche, J. Magn. Magn. Mater. 2007, 310, e947.

[135] E. Ferreiro, X. M. Bendaña, J. B. González-Diaz, A. García-Martín, J. M. GarcíaMartín, A. Cebollada, G. Armelles, D. Meneses-Rodríguez, E. Muñoz-Sandoval, IEEE Trans. Magn. 2008, 44, 3303.

[136] E. Ferreiro-Vila, J. B. González-Díaz, R. Fermento, M. U. González, A. GarcíaMartín, J. M. García-Martín, A. Cebollada, G. Armelles, D. Meneses-Rodríguez, E. Muñoz-Sandoval, Phys. Rev. B 2009, 80, 125132.

[137] E. Ferreiro-Vila, M. Iglesias, E. Paz, F. J. Palomares, F. Cebollada, J. M. González, G. Armelles, J. M. García-Martín, A. Cebollada, Phys. Rev. B 2011, 83, 205120. 
[138] K. Yang, C. Clavero, J. R. Skuza, M. Varela, R. A. Lukaszew, J. Appl. Phys. 2010, 107, 103924.

[139] V. G. Kravets, A. S. Lapchuk, Appl. Opt. 2010, 49, 5013.

[140] D. M. Newman, M. L. Wears, R. J. Matelon, I. R. Hooper, J. Phys.: Condens. Matter 2008, 20, 345230.

[141] A. A. Grunin, A. G. Zhdanov, A. A. Ezhov, E. A. Ganshina, A. A. Fedyanin, Appl. Phys. Lett. 2010, 97, 261908.

[142] C. Clavero, K. Yang, J. R. Shuza, R. A. Lukaszew, Opt. Lett. 2010, 35, 1557.

[143] J. Bremer, V. Vaicikauskas, F. Hansteen, O. Hunderi, J. Appl. Phys. 2001, 89, 6177.

[144] V. E. Kochergin, A. Y. Toporov, M. V. Valeiko, J. Exp. Theor. Phys. Lett. 1998, $68,400$.

[145] V. Kochergin, P. R. Swinehart, in Magneto-optical Imaging, (Eds: T. H. Johansen, D. V. Shantsev), Kluwer Academic Publishers, The Netherlands 2004, p. 337. [146] D. M. Newman, M. Wears, R. Matelon, J. Magn. Magn. Mater. 2002, 242-245 Part 2, 980.

[147] V. V. Pavlov, G. Tessier, C. Malouin, P. Georges, A. Brun, D. Renard, P. Meyer, J. Ferré, P. Beauvillain, Appl. Phys. Lett. 1999, 75, 190.

[148] V. K. Valev, A. V. Silhanek, W. Gillijns, Y. Jeyaram, H. Paddubrouskaya, A. Volodin, C. G.Biris, N. C. Paoiu, B. D. Clercq, M. Ameloot, O. A. Aktsipetrov, V. V. Moshchalkov, T. Verbiest, ACS Nano 2011, 5, 91.

[149] T. A. Kelf, Y. Sugawara, R. M. Cole, J. J. Baumberg, M. E. Abdelsalam, S. Cintra, S. Mahajan, A. E. Russell, P. N. Bartlett, Phys. Rev. B 2006, 74, 245415.

[150] M. Diwekar, V. Kamaev, J. Shi, Z. V. Vardeny, Appl. Phys. Lett. 2004, 84, 3112. 
[151] G. Ctistis, E. Papaioannou, P. Patoka, J. Gutek, P. Fumagalli, M. Giersig, Nano Lett. 2009, 9, 1.

[152] E. Th. Papaioannou, V. Kapaklis, P. Patoka, M. Giersig, P. Fumagalli, A. GarcíaMartín, E. Ferreiro-Vila, Phys. Rev. B 2010, 81, 054424.

[153] J. F. Torrado, E. Th. Papaioannou, G. Ctistis, P. Patoka, M. Giersig, G. Armelles, A. García-Martín, Phys. Status Solidi RRL 2010, 4, 271.

[154] J. B. González-Díaz, J. M. García-Martín, A. García-Martín, D. Navas, A. Asenjo, M. Vázquez, M. Hernández-Vélez, G. Armelles, Appl. Phys. Lett. 2009, 94, 263101.

[155] E. Th. Papaioannou, V. Kapaklis, E. Melander, B. Hjörvarsson, S. D. Pappas, P. Patoka, M. Giersig, P. Fumagalli, A. García-Martín, G. Ctistis, Opt. Express 2011, 19, 23867.

[156] Z- Liu, L. Shi, Z. Shi, X. H. Liu, J. Zi, S. M. Zhou, S. J. Wei, J. Li, Z. Zhang, Appl. Phys. Lett. 2009, 95, 032502.

[157] M. V. Sapozhnikov, S. A. Gusev, V. V. Rogov, O. L. Ermolaeva, B. B. Troitskii, L. V. Khokhlova, D. A. Smirnov, Appl. Phys. Lett. 2010, 96, 122507.

[158] M. V. Sapozhnikov, S. A. Gusev, B. B. Troitskii, L. V. Khokhlova, Opt. Lett. 2011, 36, 4197.

[159] J. F. Torrado, J. B. González-Díaz, G. Armelles, A. García-Martín, A. Altube, M. López-García, J. F. Galisteo-López, A. Blanco, C. López, Appl. Phys. Lett. 2011, 99, 193109.

[160] A. A. Grunin, N. A. Sapoletova, K. S. Napolskii, A. A. Eliseev, A. A. Fedyanin, J. Appl. Phys. 2012, 111, 07A948.

[161] A. V. Chetvertukhin, A. V. Baryshev, H. Uchida, M. Inoue, A. A. Fedyanin, J. Appl. Phys. 2012, 111, 07A946. 
[162] M. Rubio-Roy, O. Vlasin, O. Pascu, J. M. Caicedo, M. Schmidt, A. R. Goñi, N.

G. Tognalli, A. Fainstein, A. Roig, G. Herranz, Langmuir 2012, 28, 9010.

[163] Y. M. Strelniker, D. J. Bergman Phys. Rev. B 1999, 59, R12763.

[164] Y. M. Strelniker, D. J. Bergman, Phys. Rev. B 2008, 77, 205113.

[165] A. Battula, S. Chen, Y. Lu, R. J.Knize, K. Reinhardt, Opt. Lett. 2007, 18, 2692.

[166] V. I. Belotelov, L. L. Doskolovich, A. K. Zvezdin, Phys. Rev. Lett. 2007, 98, 077401.

[167] V. I. Belotelov, D. A. Bykov, L. L. Doskolovich, A. N. Kalisch, V. A. Kotov, A. K. Zvezdin, Opt. Lett. 2009, 34, 398.

[168] V. I. Belotelov, I. A. Akimov, M. Pohl, V. A. Kotov, S. Kasture, A. S. Vengurlekar, A. V. Gopal, D. R. Yakovlev, A. K. Zvezdin, M. Bayer, Nat. Nanotechnol. 2011, 6, 370.

[169] G. A. Wurtz, W. Hendren, R. Pollard, R. Atkinson, L. Le Guyader, A. Kirilyuk, Th Rasing, I. I. Smolyaninov, A. V. Zayats, New J. Phys. 2008, 10, 105012.

[170] L. P. Pitaevskii, Sov. Phys. JETP 1961, 12, 1008.

[171] P. S. Pershan, Phys. Rev. 1963, 130, 919.

[172] J. P. van der Ziel, P. S. Pershan, L. D. Malmstrom, Phys. Rev. Lett. 1965, 15, 190.

[173] I. I. Smolyaninov, C. C. Davis, V. N. Smolyaninova, D. Schaefer, J. Elliott, A. V. Zayats, Phys. Rev. B 2005, 71, 035425.

[174] Y. Gu, K. G. Kornev, J. Opt. Soc. Am. B 2010, 27, 2165.

[175] V. I. Belotelov, E. A. Bezus, L. L. Doskolovich, A. N. Kalish, A. K. Zvezdin, J. Phys.: Conf. Ser. 2010, 200, 092003.

[176] V. I. Belotelov, A. K. Zvezdin, arXiv:1112.0681 2011.

[177] G. Weick, D. Weinmann, Phys. Rev. B 2011, 83, 125405. 
[178] R. E. De Wames, W. F. Hall, Phys. Rev. Lett. 1972, 29, 172.

[179] A. Hartstein, E. Burstein, A. A. Maradudin, R. Brewer, R. F. Wallis, J. Phys. C: Solid State Phys. 1973, 6, 1266.

[180] R. F. Wallis, J. J. Brion, E. Burstein, A. Hartstein, Phys. Rev. B 1974, 9, 3424.

[181] Y. O. Nakamura, B. V. Paranjape, Solid State Commun. 1975, 16, 467.

[182] When the dielectric is the magnetic medium, with optical and MO constants $\varepsilon_{d}$ and $\varepsilon_{m o}$, respectively, and the metal is characterized by $\varepsilon_{m}$, the expression of $\Delta \mathrm{k}_{\mathrm{sp}}$ is modified as follows: $\Delta k_{s p}=\frac{k_{s p}^{0} \varepsilon_{m o}}{\sqrt{\varepsilon_{d} \varepsilon_{m}}\left(1-\frac{\varepsilon_{d}^{2}}{\varepsilon_{m}^{2}}\right)}$.

[183] D. Sarid, Phys. Rev. B 1984, 29, 2344.

[184] M. S. Kushwaha, P. Halevi, Phys. Rev. B, 1987, 35, 3879.

[185] M. S. Kushwaha, P. Halevi, Phys. Rev. B 1987, 36, 5960.

[186] M. S. Kushwaha, P. Halevi, Phys. Rev. B 1988, 38, 12428.

[187] R.K. Hickernell, D. Sarid, Opt. Lett. 1987, 12, 570.

[188] Y.-C. Lan, Y.-C. Chang, P.-H. Lee, Appl. Phys. Lett. 2007, 90, 171114.

[189] Y.-C. Lan, C.-M. Chen, Opt. Express 2010, 18, 12470.

[190] X.-X. Liu, C.-F. Tsai, R.-L. Chern, D. P. Tsai, Appl. Opt. 2009, 48, 3102.

[191] D. M. Newman, M. L. Wears, R. J. Matelon, Europhys. Lett. 2004, 68, 692.

[192] D. Martín-Becerra, V. V. Temnov, T. Thomay, A. Leitenstorfer, R. Bratschitsch, G. Armelles, A. García-Martín, M. U. González, Phys. Rev. B 2012, 86, 035118.

[193] D. Martín-Becerra, J. B. González-Díaz, V. V. Temnov, A. Cebollada, G. Armelles, T. Thomay, A. Leitenstorfer, R. Bratschitsch, A. García-Martín, M. U. González, Appl. Phys. Lett. 2010, 97, 183114. 
[194] H. Ditlbacher, A. Hohenau, D. Wagner, U. Kreibig, M. Rogers, F. Hofer, F. R. Aussenegg, J. R. Krenn, Phys. Rev. Lett. 2005, 95, 257403.

[195] V. A. Fedotov, T. Uchino, J. Y. Ou, Opt. Express 2012, 20, 9545.

[196] M. Kuttge, E. J. R. Vesseur, J. Verhoeven, H. J. Lezec, H. A. Atwater, A. Polman, Appl. Phys. Lett. 2008, 93, 113110.

[197] J.F. Torrado, J.B. González-Díaz, M.U. González, A. García-Martín, G. Armelles, Opt. Express 2010, 18, 15635.

[198] A. Christ, T. Zentgraf, S. G. Tihodeev, N. A. Gippius, J. Kuhl, H. Giessen, Phys. Rev. B 2006, 74, 155435.

[199] Y. Chu, K. B. Crozier, Opt. Lett. 2009, 34, 244.

[200] R. E. Camley, Surf. Sci. Rep. 1987, 7, 103.

[201] V. Zayets, H. Saito, S. Yuasa, K. Ando, J. Appl. Phys. 2012, 111, 023103.

[202] F. G. Elmzughi, D. R. Tilley, J. Phys.: Condens. Matter 1994, 6, 4233.

[203] B. L. Johnson, H.-H. Shiau, J. Phys.: Condens. Matter 2008, 20, 335217.

[204] T. Tsurumi, J. Phys. Soc. Jpn. 2007, 76, 064005.

[205] A. Boardman, N. King, Y. Rapoport, L. Velasco, New J. Phys. 2005, 7, 191.

[206] A. B. Khanikaev, S. H. Mousavi, G. Shvets, Y. S. Kivshar, Phys. Rev. Lett. 2010, 105,126804

[207] G. Düchs, G.L.J.A. Rikken, T.Grenet, P.Wyder, Phys. Rev. Lett. 2001, 87, 127402.

[208] D. Regatos, Biosensores ópticos de alta sensibilidad basados en técnicas de modulación plasmónica, PhD Thesis, Universidade de Santiago de Compostela 2012.

[209] K. Kämpf , S. Kübler , F. W. Herberg, A. Ehresmann, J. Appl. Phys. 2012, 112, 034505 .

[210] J. B. Khurgin, Appl. Phys. Lett. 2006, 89, 251115. 
[211] V. Kuzmiak, S. Eyderman, M. Vanwolleghem, Phys. Rev. B 2012, 86, 045403.

[212] J. Montoya, K. Parameswaran, J. Hensley, M. Allen, R. Ram, J. Appl. Phys. 2009, 106, 023108.

[213] M. Vanwolleghem, W. Van Parys, D. Van Thourhout, R. Baets, F. Lelarge, O. Gauthier-Lafaye, B. Thedrez, R. Wirix-Speetjens, L. Lagae, Appl. Phys. Lett. 2004, 85, 3980.

[214] W. Van Parys, B. Moeyersoon, D. Van Thourhout, R. Baets, M. Vanwolleghem, B. Dagens, J. Decobert, O. Le Gouezigou, D. Make, R. Vanheertum, L. Lagae, Appl. Phys. Lett. 2006, 88, 071115.

[215] H. Shimizu, Y. Nakano, Jpn. J. Appl. Phys. 2004, 43, L1561.

[216] H. Shimizu, Y. Nakano, J. Lightwave Technol. 2006, 24, 38.

[217] B. K. Chau, S. Irvine, A. Elezzabi, IEEE J. Quantum Electron. 2004, 40, 571.

[218] W. Chen, N. Xu, L. Xu, L. Wang, Z. Li, W. Ma, Y. Zhu, C. Xu, N. A. Kotov, Macromol. Rapid Commun. 2010, 31, 228.

[219] F. M. Kievit, M. Zhang, Acc. Chem. Res. 2011, 44, 853.

[220] X. Huang, I. H. El-Sayed, W. Qian, M. A. El-Sayed, J. Am. Chem. Soc. 2006, $128,2115$.

[221] P. K. Jain, X. Huang, I. H. El-Sayed, M. A. El-Sayed, Acc. Chem. Res. 2008, 41, 1578.

[222] R. Bardhan, S. Lal, A. Joshi, N. J. Halas, Acc. Chem. Res. 2011, 44, 936.

[223] J. Gao, H. Gu, B. Xu, Acc. Chem. Res. 2009, 42, 1097.

[224] J. Xie, G. Liu, H. S. Eden, H. Ai, X. Chen, Acc. Chem. Res. 2011, 44, 883.

[225] C. Tassa, S. Y. Shaw, R. Weissleder, Acc. Chem. Res. 2011, 44, 842.

[226] M. Famulok, J. S. Hartig, G. Mayer, Chem. Rev. 2007, 107, 3715. 
[227] S. Schrittwieser, F. Ludwig, J. Dieckhoff, K. Soulantica, G. Viau, L.-M. Lacroix, S. Mozo Lentijo, R. Boubekri, J. Maynadié, A. Huetten, H. Brueckl, J. Schotter, ACS Nano 2012, 6, 791.

[228] Y. Zhang, D. DaSilva, B. Ashall, G. Doyle, D. Zerulla, T. D. Sands, G. U. Lee, Langmuir 2011, 27, 15292.

[229] Y. Zhang, Q. Wang, Adv. Mater. 2012, 24, 2485.

[230] C. de Julián Fernández, G. Mattei, E. Paz, R. L. Novak, L. Cavigli, L. Bogani, F. J. Palomares, P. Mazzoldi, A. Caneschi, Nanotechnology 2010, 21, 165701.

[231] L. Bogani, L. Cavigli, C. de Julián Fernández, P. Mazzoldi, G. Mattei, M. Gurioli, M. Dressel, D. Gatteschi, Adv. Mater. 2010, 22, 4054.

[232] F. H. L. Koppens, D. E. Chang, F. J. García de Abajo, Nano Lett. 2011, 11, 3370. [233] L. Ju, B. Geng, J. Horng, C. Girit, M. Martin, Z. Hao, H. A. Bechtel, X. Liang, A. Zettl, Y. R. Shen, F. Wang, Nat. Nanotech. 2011, 6, 630.

[234] I. Crassee, J. Levallois, A. L. Walter, M. Ostler, A. Bostwick, E. Rotenberg, T. Seyller, D. van der Marel, A. B. Kuzmenko, Nat. Phys. 2011, 7, 48.

[235] I. Crassee, M. Orlita, M. Potemski, A. L. Walter, M. Ostler, Th. Seyller, I. Gaponenko, J. Chen, A. B. Kuzmenko, Nano Lett. 2012, 12, 2470.

[236] H. Yan, Z. Li, X. Li, W. Zhu, P. Avouris, F. Xia, Nano Lett. 2012, 12, 3766.

[237] M. Tonouchi, Nat. Photon. 2007, 1, 97.

[238] S. A. Maier, S. R. Andrews, L. Martín-Moreno, F. J. García-Vidal, Phys. Rev. Lett. 2006, 97, 176805.

[239] W. Zhu, A. Agrawal, A. Nahata, Opt. Express 2008, 16, 6216.

[240] D. Martin-Cano, M. L. Nesterov, A. I. Fernandez-Dominguez, F. J. Garcia-Vidal, L. Martin-Moreno, E. Moreno, Opt. Express 2010, 18, 754.

[241] B. Hu, Q. J. Wang, S. W. Kok, Y. Zhang, Plasmonics 2012, 7, 191. 
[242] B. Hu, Q. J. Wang, Y. Zhang, Opt. Lett. 2012, 37, 1895. 


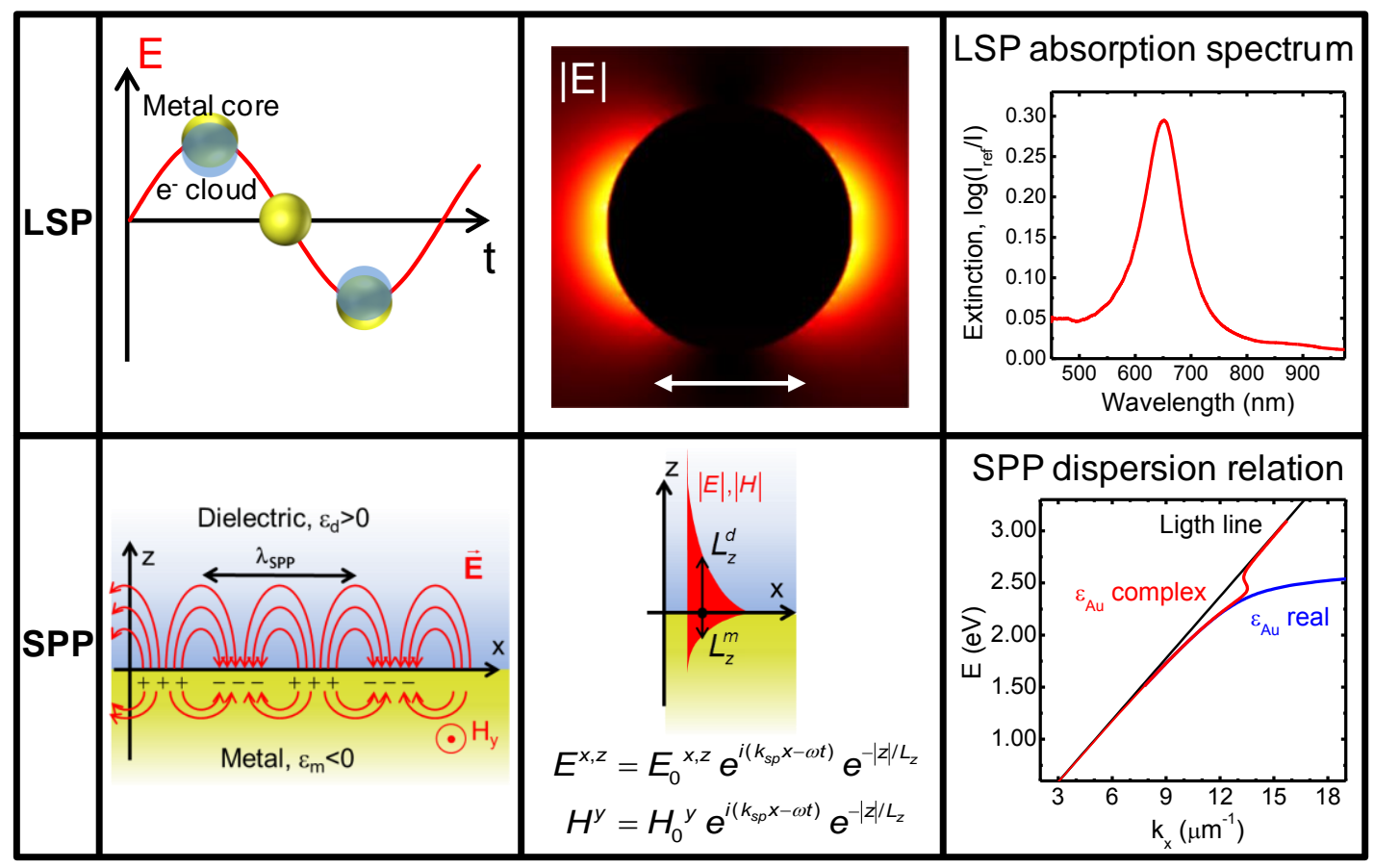

Figure 1. Top: (left) Sketch of charges oscillation for a localized surface plasmon (LSP) in a metallic nanoparticle; (center) Calculated field distribution for a Au nanodisk under LSP excitation; (right) Extinction spectrum of a set of Au nanodisks (120 nm diameter, $40 \mathrm{~nm}$ height) on glass. Bottom: (left) Sketch of charges oscillation and fields for a surface plasmon polariton (SPP) in a metal/dielectric interface; (center) Evanescent SPP field distribution; (right) Dispersion relation of a Au/air SPP, considering both the cases of $\mathrm{Au}$ without absorption (real dielectric permittivity, blue line), and with absorption (complex dielectric permittivity, red line). 


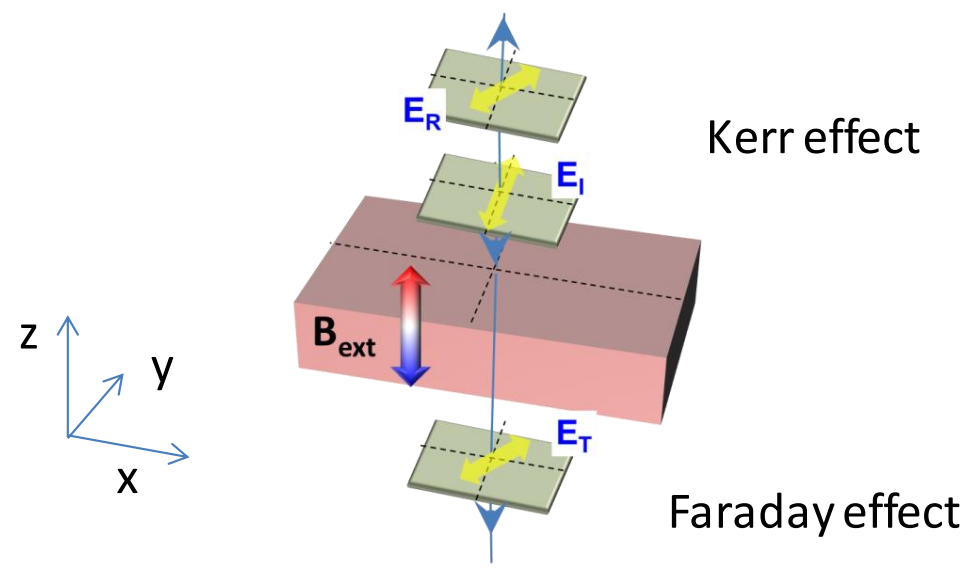

\begin{tabular}{|c|c|c|}
\hline \multicolumn{3}{|c|}{ Kerr effect } \\
\hline Polar & Longitudinal & Transversal \\
\hline$\left(\begin{array}{ccc}\varepsilon & a \Pi & 0 \\
-a \Pi & \varepsilon & 0 \\
0 & 0 & \varepsilon\end{array}\right)$ & $\left(\begin{array}{ccc}\varepsilon & 0 & 0 \\
0 & \varepsilon & -a \Pi \\
0 & a \Pi & \varepsilon\end{array}\right)$ & $\left(\begin{array}{ccc}\varepsilon & 0 & a \Pi \\
0 & \varepsilon & 0 \\
-a \Pi & 0 & \varepsilon\end{array}\right)$ \\
\hline$\left(\begin{array}{cc}r_{p p} & r_{p s}(\Pi) \\
r_{s p}(\Pi) & r_{s s}\end{array}\right)$ & $\left(\begin{array}{cc}r_{p p} & r_{p s}(\Pi) \\
r_{s p}(\Pi) & r_{s s}\end{array}\right)$ & $\left(\begin{array}{cc}r_{p p}(\Pi) & 0 \\
0 & r_{s S}\end{array}\right)$ \\
\hline$\theta+i \varphi=\frac{r_{p S}(\Pi)}{r_{p p}}$ & $\theta+i \varphi=\frac{r_{p s}(\Pi)}{r_{p p}}$ & $\frac{\Delta R_{p p}(\Pi)}{R_{p p}}$ \\
\hline
\end{tabular}

Figure 2. Top drawing: Sketch illustrating the magneto-optical (MO) effects, both in reflection (Kerr effect) and in transmission (Faraday effect). Bottom panel: The three possible MO configurations, defined regarding the relative orientation between the magnetic field and the plane of incidence, illustrated for the Kerr effect. The four rows in the pannel provide, respectively: the configuration geometry, the corresponding dielectric tensor, the associated reflectivity matrix and the relevant measurable quantities. 

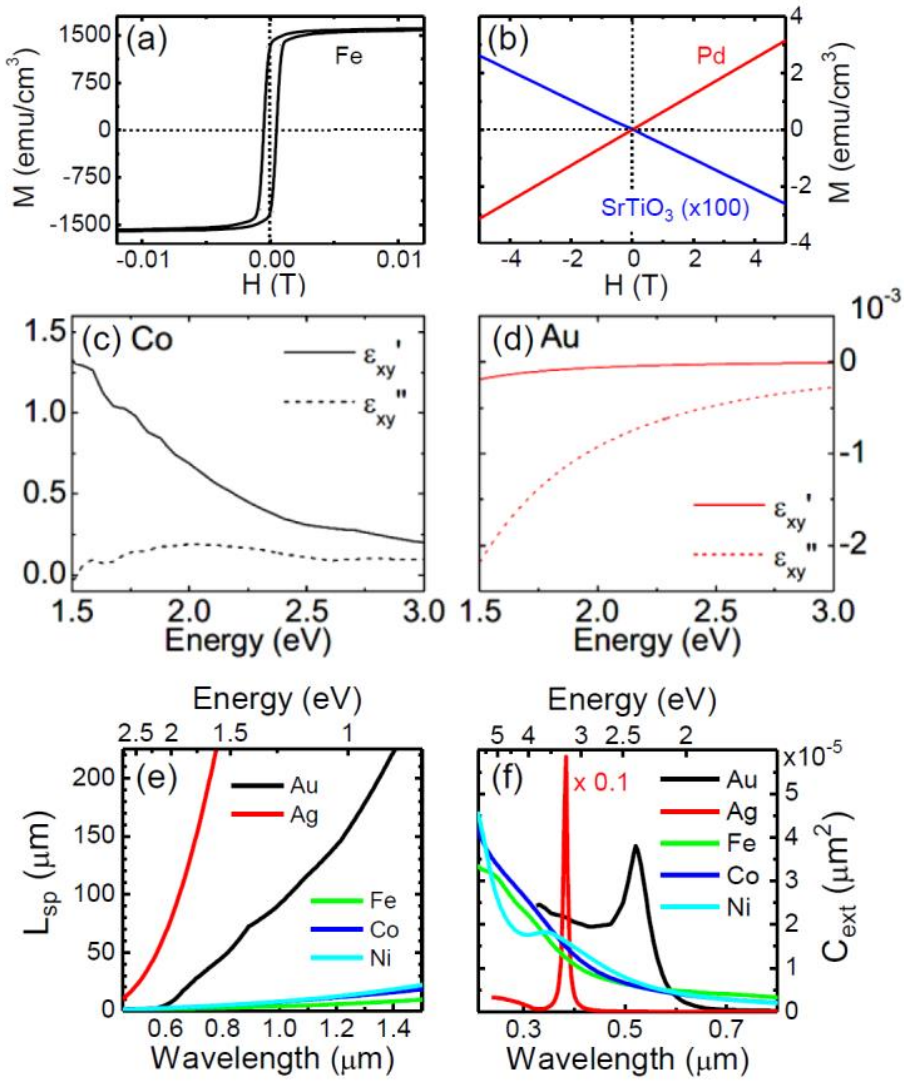

Figure 3. Magnetic response for a ferromagnetic material, $\mathrm{Fe}(\mathrm{a})$ and a diamagnetic and a paramagnetic material, $\mathrm{SrTiO}_{3}$ and $\mathrm{Pd}$ respectively (b). Real and imaginary part of the MO constants of $\mathrm{Co}(\mathrm{c})$ and $\mathrm{Au}$ (d). For Co, these constants were experimentally obtained, whereas those for $\mathrm{Au}$ were obtained through a Drude model calculation considering an applied magnetic field of 1 T. (e): Calculated SPP propagation distance, $\mathrm{L}_{\mathrm{sp}}$, for noble and ferromagnetic metals. (f): Calculated extinction cross-section for 10nm-diameter spheres of noble and ferromagnetic metals in water. The optical constants for $\mathrm{Au}$ and $\mathrm{Ag}$ were taken from Ref. [69], and those for ferromagnetic metals from Ref. [70]. (c)-(d) are reproduced with permission from [107]. Copyright 2010, American Institute of Physics. 

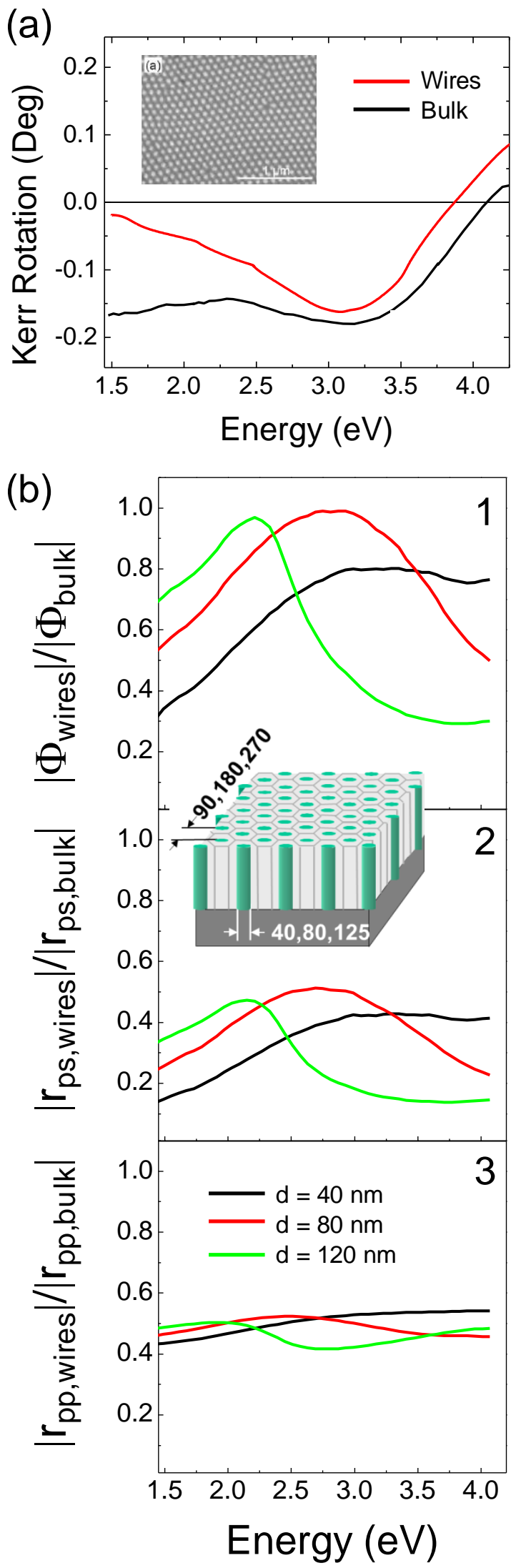

Figure 4. (a) SEM image of an hexagonal array of nickel nanowires embedded in an alumina matrix. The nanowire diameter is $40 \mathrm{~nm}$ and the interwire distance, $105 \mathrm{~nm}$. 
The red curve represents the Kerr rotation spectrum of the array shown in the SEM image and the black curve represents the Kerr rotation spectrum of a nickel thick film. Due to the excitation of the LSP resonance of the wires, an enhancement of the rotation around $3 \mathrm{eV}$ is clearly observed. (b) Calculated curves of: modulus of the complex Polar Kerr rotation, $\Phi(1)$, modulus of $r_{p s}(2)$ and modulus of $r_{p p}(3)$ for arrays of Ni nanowires embedded in an alumina matrix, normalized to that of bulk $\mathrm{Ni}$. The three arrays have the same amount of $\mathrm{Ni}(17 \%)$, but different nanowire diameter: $40 \mathrm{~nm}$ (black curve), $80 \mathrm{~nm}$ (red curve) and $120 \mathrm{~nm}$ (green curve) with lattice parameter of 90, 180 and $270 \mathrm{~nm}$ respectively. As it can be observed, the modulus of the Polar Kerr rotation has a peak whose position shifts towards lower energies as we increase the wire diameter. From the two contributions to the enhancement of the Kerr rotation, $r_{p s}$ and $r_{p p}$, it can be seen that the MO component localizes the enhancement in the spectral region of the LSP excitation, whereas the overall reduction of the reflectivity contributes to the enhancement in an almost wavelength independent way. Adapted with permission from [86]. 


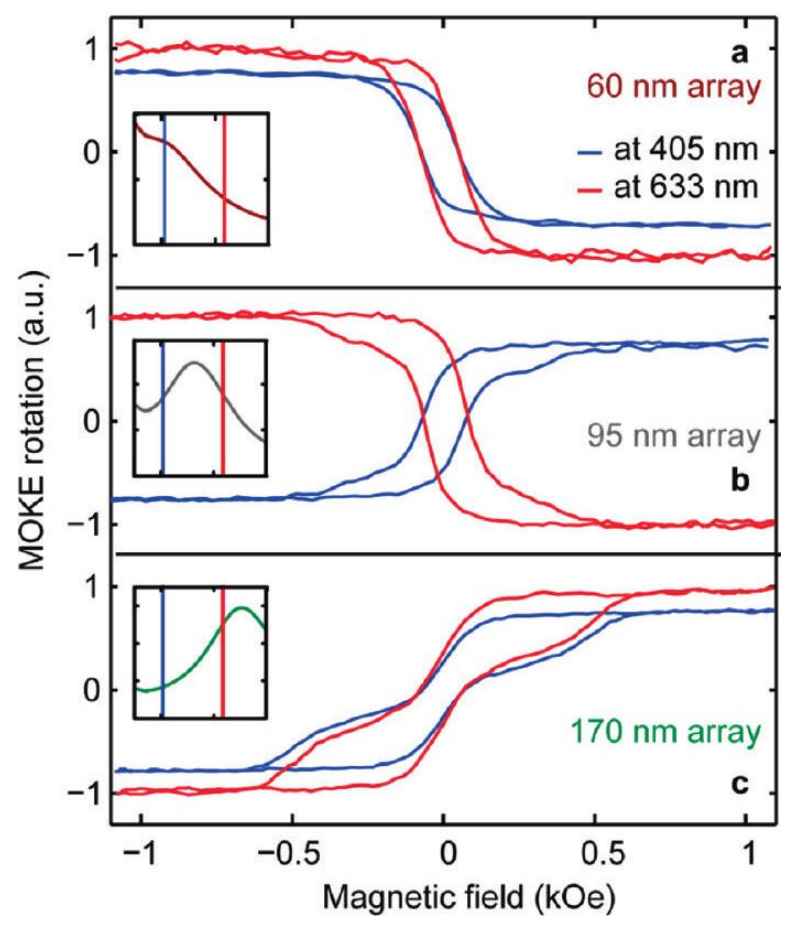

Figure 5. Normalized longitudinal MO Kerr rotation for randomly distributed nickel nanodisks of 60 (a), 95 (b), and $170 \mathrm{~nm}$ diameter (c), at two different excitation wavelengths: $405 \mathrm{~nm}$ - blue lines and $633 \mathrm{~nm}$ - red lines. The insets show the far-field extinction spectra for the corresponding nanodisks, with the blue and red line pointing the relative position of the wavelengths of the MO Kerr measurements with respect of the LSP peak. MO Kerr loops taken at the right of the LSP peaks have an opposite sign to those taken at the left due to the change of sign in the particles polarizability when crossing the LSP resonance. Reproduced with permission from [89]. Copyright 2011, American Chemical Society. 

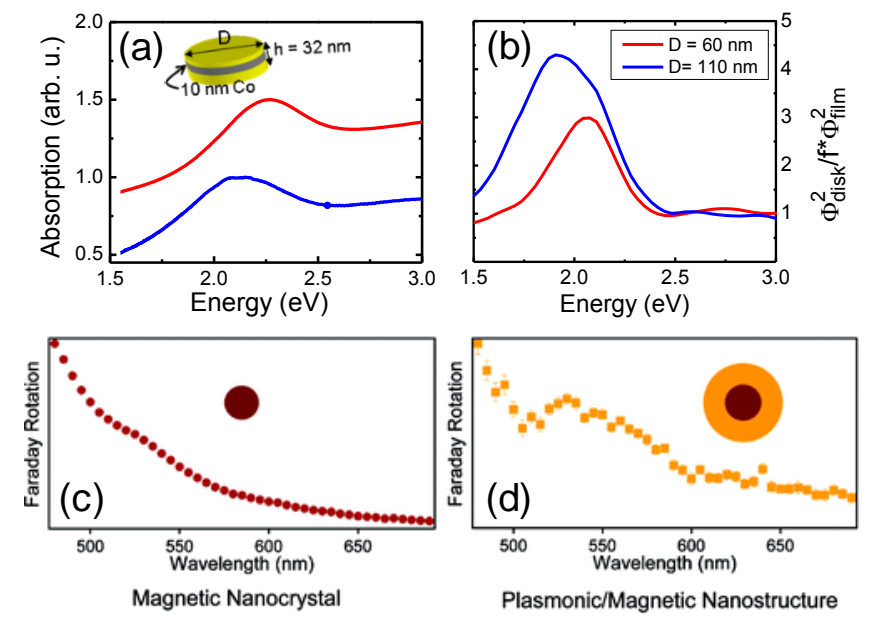

- ${ }_{\gamma} \mathrm{Fe} 2 \mathrm{O} 3=\mathrm{Au}$

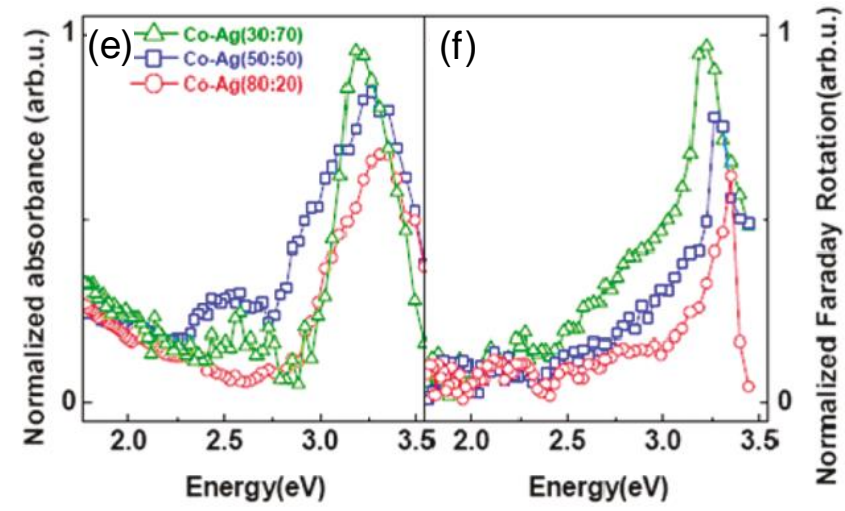

Figure 6. (a) Absorption spectra of $\mathrm{Au} / \mathrm{Co} / \mathrm{Au}$ nanodisks of diameter 60 and $110 \mathrm{~nm}$, showing the presence of an LSP resonance. (b) Ratio of the squared modulus of the complex Polar Kerr rotation for the nanodisks normalized to that of an equivalent continuous $\mathrm{Au} / \mathrm{Co} / \mathrm{Au}$ film and weighted by the nanodisks concentration. (c) Faraday rotation spectrum of $\gamma-\mathrm{Fe}_{2} \mathrm{O}_{3}$ nanoparticles and (d) same for gold-coated $\gamma-\mathrm{Fe}_{2} \mathrm{O}_{3}$ nanoparticles, showing the effect of the LSP resonance of the Au shell. (e) Absorption and (f) Faraday rotation spectra for $\mathrm{Co}-\mathrm{Ag}$ nanoparticles with different $\mathrm{Co}: \mathrm{Ag}$ concentration ratios. The Faraday rotation and absorption peaks decrease and blue-shift when the Co concentration increases. (a) and (b) are adapted with permission from [71]. Copyright 2009, IOP Publishing Ltd. (c)-(d) are reproduced with permission from [95]. Copyright 2009, American Chemical Society. (e)-(f) are reproduced with permission from [99]. Copyright 2011, American Chemical Society. 

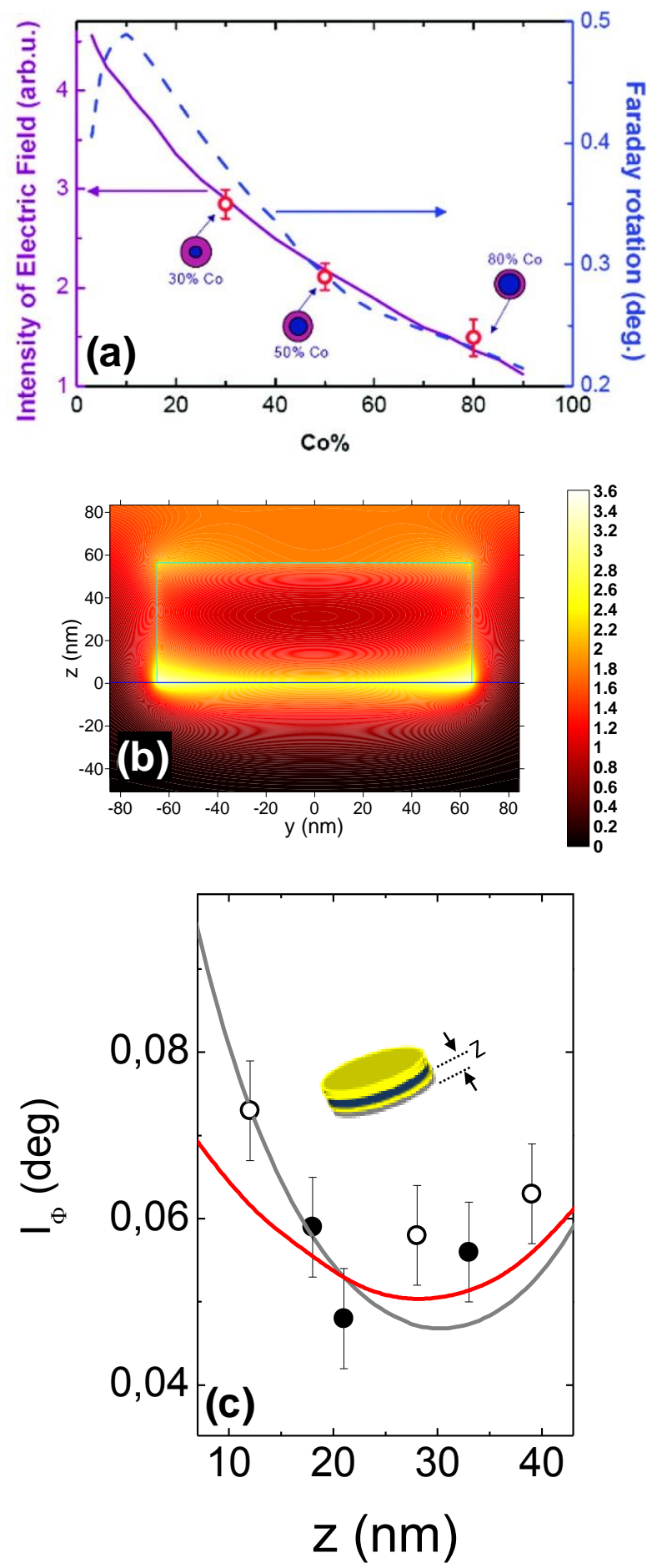

Figure 7. (a) Calculated intensity of the EM field at resonance within the Co core and maximum Faraday rotation for Co-Ag core-shell nanoparticles embedded in oil as a function of the Co concentration. The direct relation between the EM field and the MO signal is clearly observed. (b) Calculated EM field distribution at resonance for a $\mathrm{Au}$ 
nanodisk on top of a glass substrate. Note the inhomogeneous field distribution inside the nanodisk. (c) MO activity as a function of Co position, $\mathrm{z}$, for a series of $\mathrm{Au} / \mathrm{Co} / \mathrm{Au}$ nanodisks at resonance. Symbols correspond to experimental data. Full lines represent theoretical calculations of the EM field distribution within the disk structure, red line for pure $\mathrm{Au}$ disks and grey line for $\mathrm{Au}$ disks with a $6 \mathrm{~nm}$ Co layer inside at different positions. (a) is reproduced with permission from [99]. Copyright 2011, American Chemical Society. (b) and (c) are reproduced with permission from [79]. 


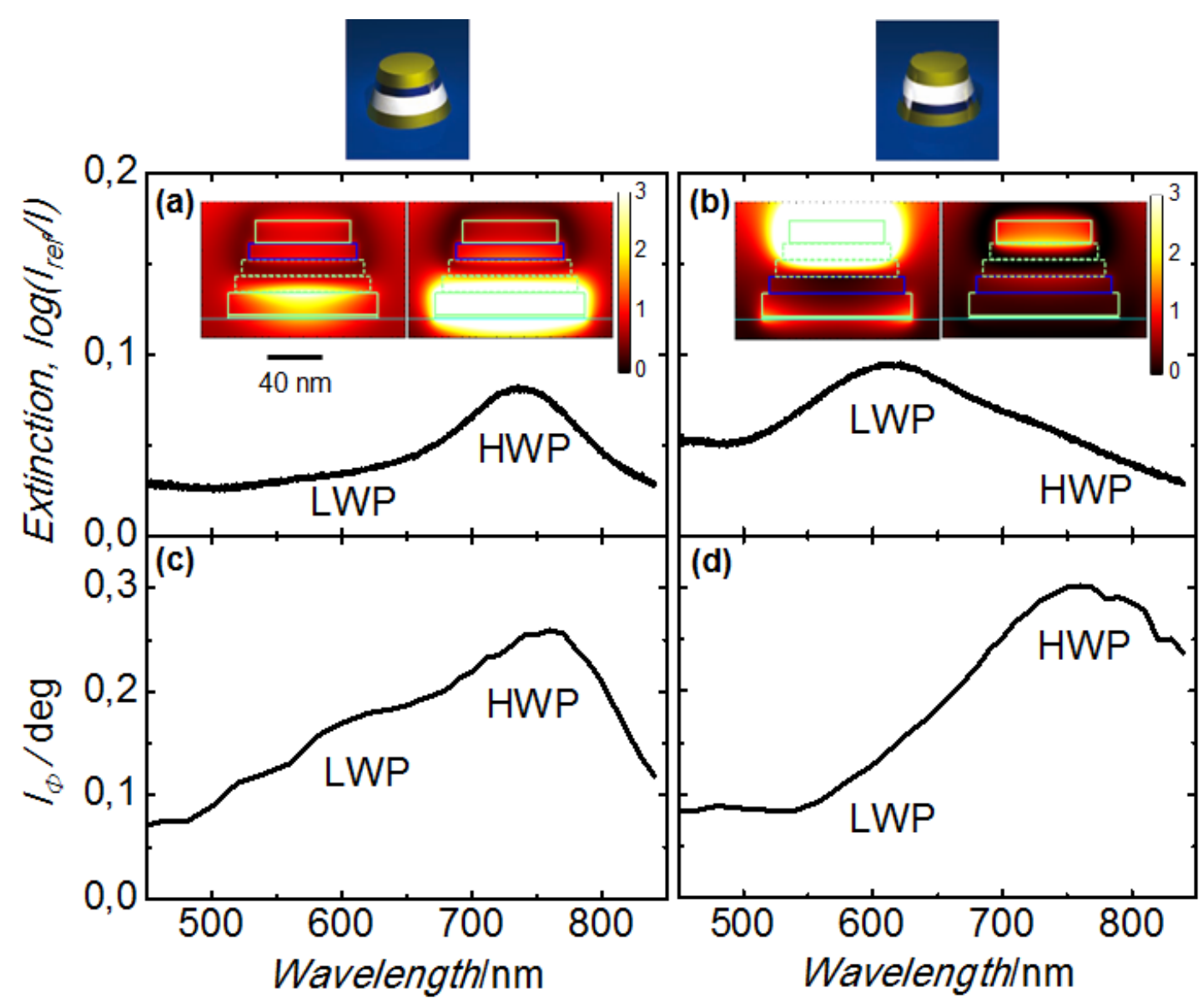

Figure 8. Extinction spectra of (a) $\mathrm{Au} / \mathrm{Co} / \mathrm{SiO}_{2} / \mathrm{Au}$ nanodisks on glass and (b) $\mathrm{Au} / \mathrm{SiO}_{2} / \mathrm{Co} / \mathrm{Au}$ nanodisks on glass. The insets represent the electromagnetic field intensity distribution within the nanodisks, showing the location of the field for the two resonance wavelengths. (c) and (d) show the spectra of the modulus of the complex Polar Kerr rotation for the two structures. Adapted with permission from [115]. 


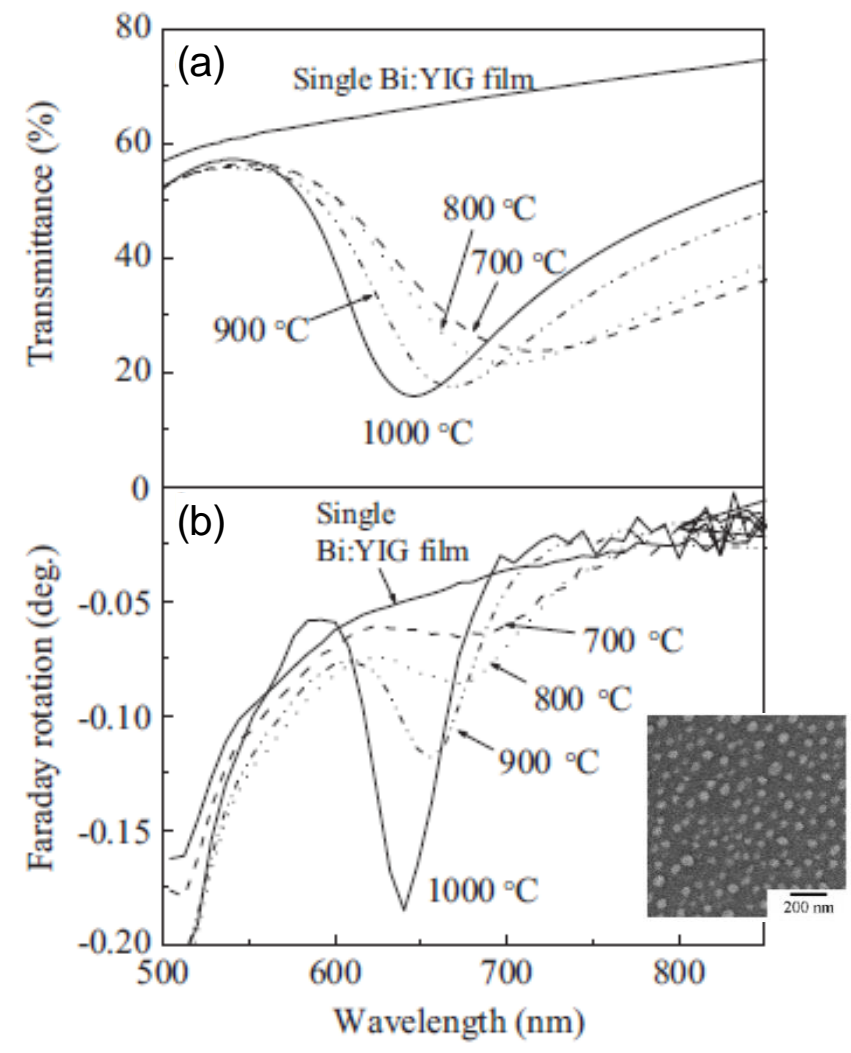

Figure 9. Transmission spectra of Au nanoparticles embedded in bismuth-substituted yttrium iron garnet (Bi:YIG). The Au nanoparticles were formed on quartz substrates by heating a $5 \mathrm{~nm}$ Au thin film. The nanoparticles were covered with a $60 \mathrm{~nm}$ thick Bi:YIG film. A clear reduction of the transmission is observed at the LSP resonance of the Au nanoparticles, whose position depends on the heating temperature. Faraday rotation spectra of the same films are also shown. A clear relationship between the LSPs and the increase in the Faraday rotation is observed. A large resonance enhancement of the Faraday rotation is obtained for the $1000{ }^{\circ} \mathrm{C}$ sample. The SEM image corresponds to the $1000{ }^{\circ} \mathrm{C}$ sample. Reproduced with permission from [120]. Copyright 2009, Elsevier B.V. 
(a)
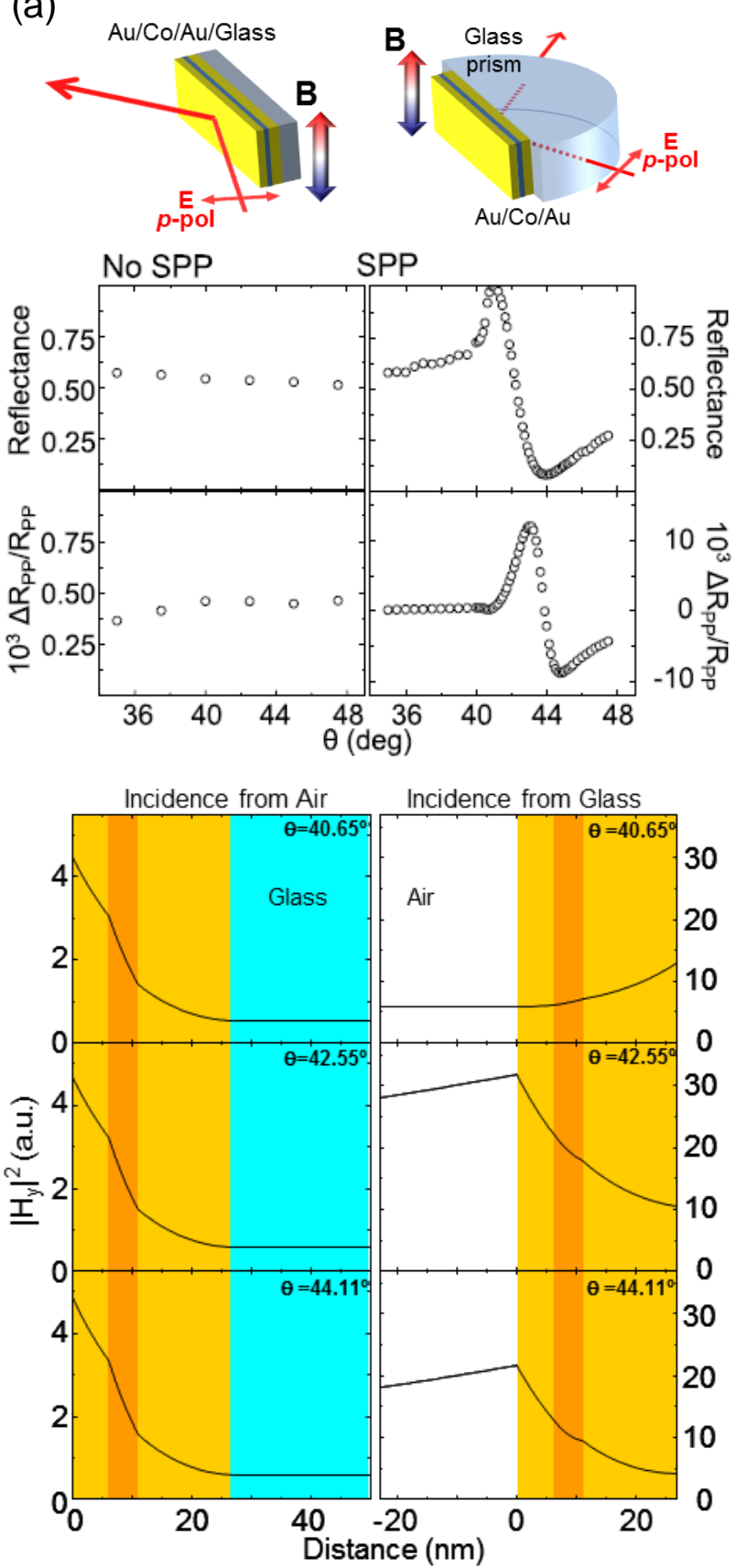

Figure 10. (a) Sketch of standard (left) and ATR (right) Kerr geometries, with an external magnetic field applied perpendicular to the plane of incidence (transversal configuration). SPP excitation is only possible under ATR geometry. Comparison between standard and ATR configurations of the reflectance (b) and the transversal MO Kerr signal (c) of a $\mathrm{Au} / \mathrm{Co} / \mathrm{Au}$ trilayer. (d) Electromagnetic (EM) field distribution inside the metallic layers for the same $\mathrm{Au} / \mathrm{Co} / \mathrm{Au}$ trilayer under standard and ATR 
configurations at three different angles. When the SPP is excited, a strong redistribution of the EM field can be seen, with a sizeable enhancement inside the Co layer.(b)-(c) are reproduced with permission from [132]. Copyright 2007, The American Physical Society. (d) is reproduced with permission from [71]. Copyright 2009, IOP Publishing Ltd. 
(a)

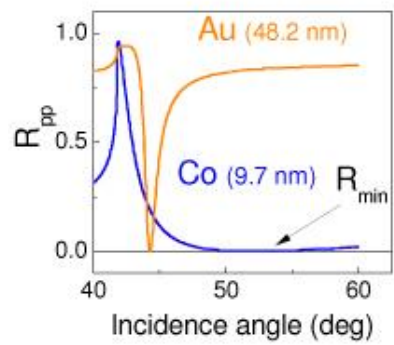

(d)

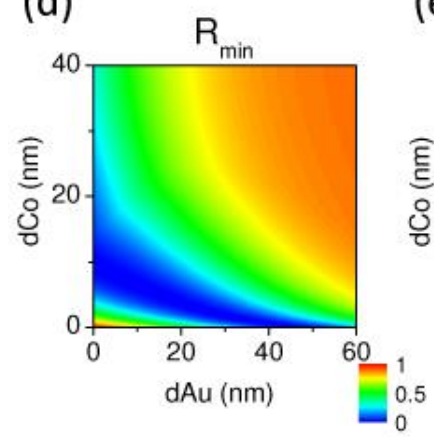

(b)

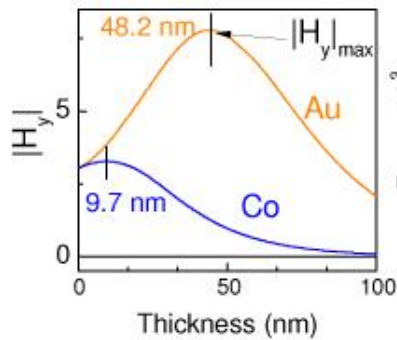

(e)

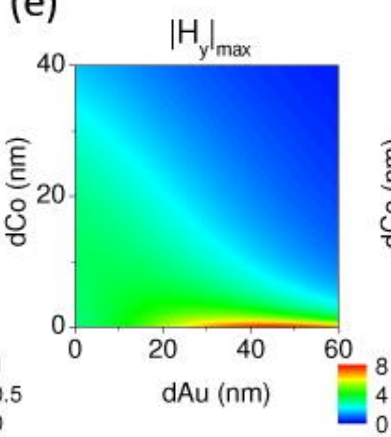

(c)

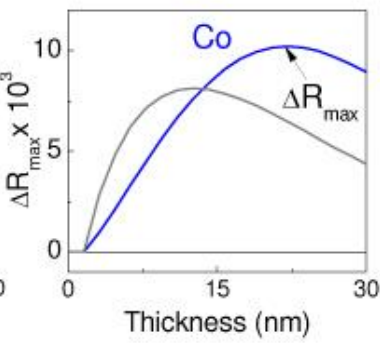

(f)

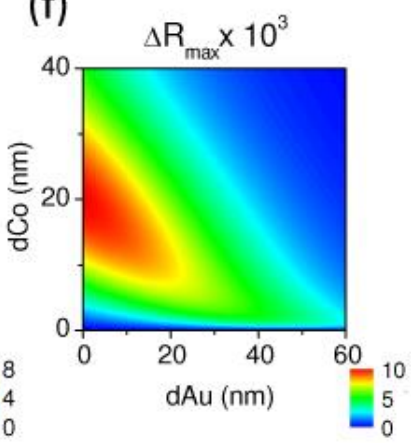

Figure 11. Calculations of: (a) reflectivity curves for $\mathrm{Co}$ and $\mathrm{Au}$ films of thickness 9.7 $\mathrm{nm}$ and $48.2 \mathrm{~nm}$ respectively; (b) SPP electromagnetic field at the metal-air interface for Co and Au films as a function of the thickness. The thickness providing maximum EM field value for each material, and therefore optimum SPP excitation, are the ones used for the reflectivity calculations in (a); (c) transversal MO Kerr effect, $\Delta R_{p p}$, for a single Co film and for $\mathrm{Au} / \mathrm{Co} / \mathrm{Au}$ trilayers (3nmAu, dnmCo, 20nmAu) as a function of the Co thickness; (d) $\mathrm{R}_{\min }$ for any combination of $\mathrm{Au}$ and Co thickness in the $\mathrm{Au} / \mathrm{Co} / \mathrm{Au}$ trilayers $(3 n m A u, d n m C o, d n m A u)$; (e) $\left|H_{y}\right|_{\max }$ for $\mathrm{Au} / \mathrm{Co} / \mathrm{Au}$ trilayers as in (d); (f) $\Delta \mathrm{R}_{\max }$ for $\mathrm{Au} / \mathrm{Co} / \mathrm{Au}$ trilayers as in (d). Reproduced with permission from [78]. Copyright 2010, Optical Society of America. 
(a)
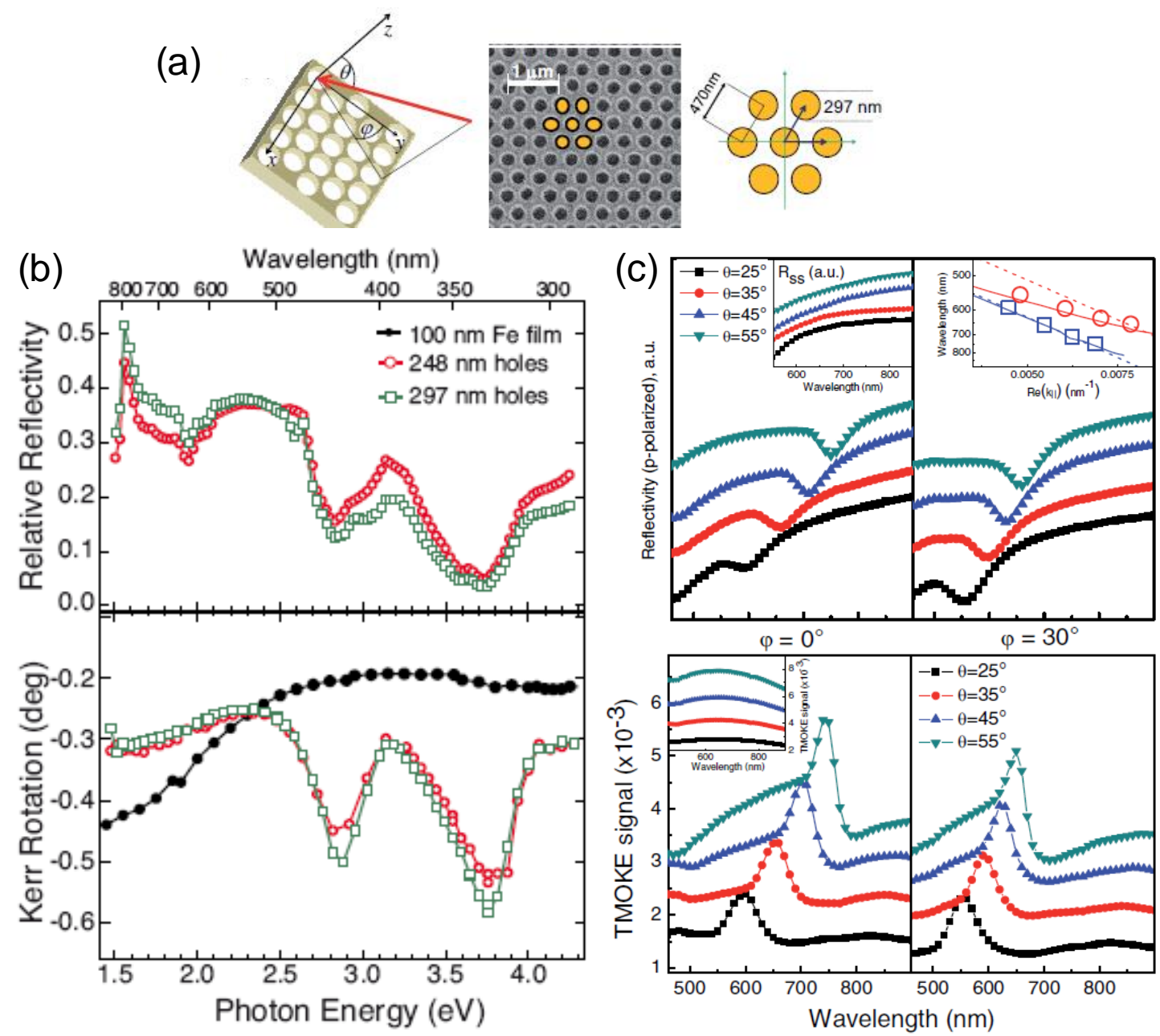

Figure 12. (a) Sketch of the perforated Fe membrane showing the definitions for the angles of incidence, a SEM of a fabricated membrane, and the geometrical parameters of the membrane. (b) Reflectivity at normal incidence (top) and the corresponding Kerr rotation (bottom) as a function of the energy of the incoming light beam for two membranes with different hole diameters. Two minima in the reflection spectra at 2.8 $\mathrm{eV}$ and $3.8 \mathrm{eV}$ attributed to plasmon excitations are seen. At the same positions, an enhancement of the Kerr rotation takes place. (c) Reflectivity (top) and transversal MO Kerr reflectivity (bottom) at off-normal incidence for two high symmetry directions of the in-plane lattice for $p$-polarized light (s-polarized in the insets). The evolution of the excitation of the low energy SPP as the incoming angle is varied can be seen, as well as how the transversal MO Kerr signal is largely enhanced at the excitation wavelengths. 
(b) is reproduced with permission from [152]. Copyright 2010, The American Physical Society. (a)-(c) are reproduced with permission from [153]. 

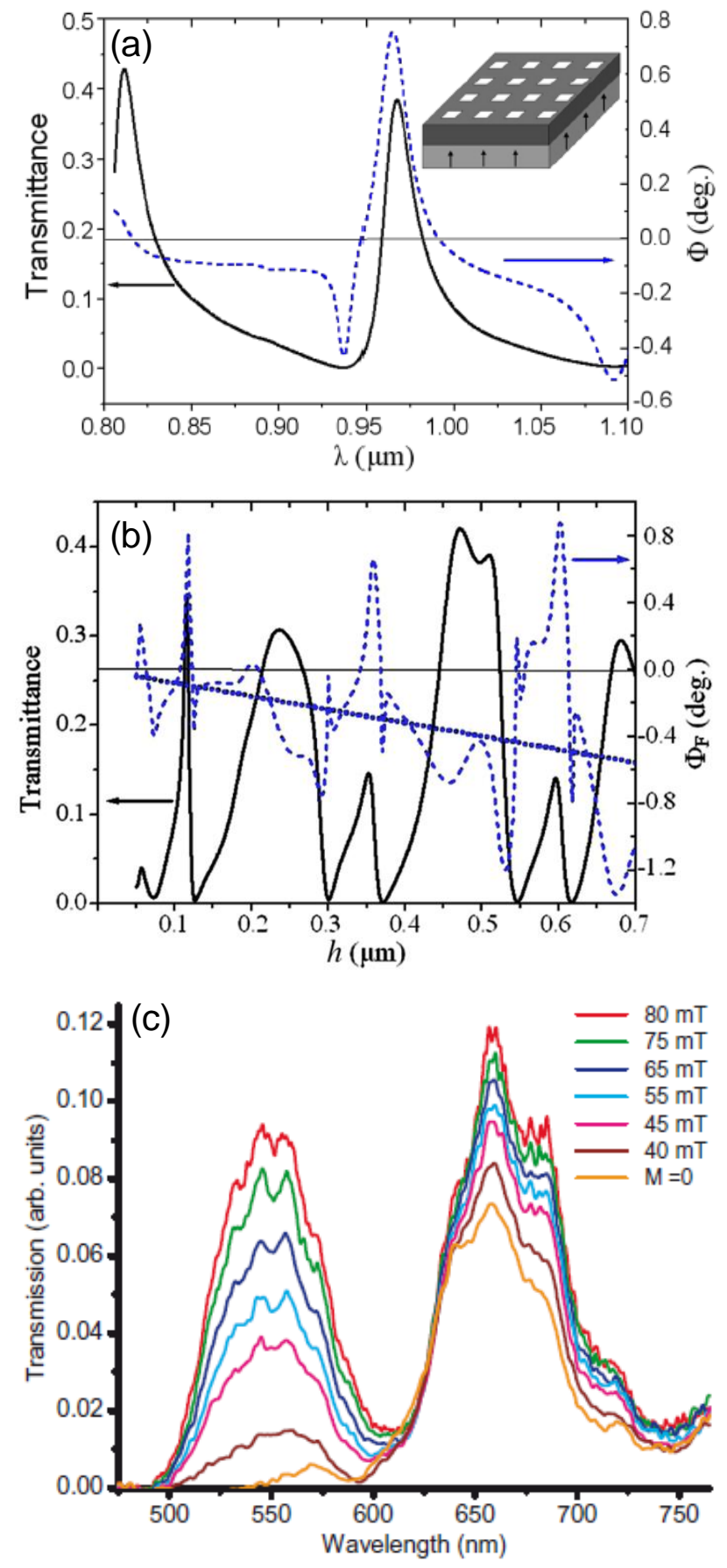

Figure 13. (a): Calculated spectra of the optical transmittance (solid line) and Faraday rotation (dashed line) for a magnetoplasmonic crystal consisting of a perforated 68-nmthick Au-film on top of a uniform 118-nm-thick bismuth-substituted yttrium iron garnet (Bi:YIG) film. The holes in the perforated membrane, of radius $395 \mathrm{~nm}$, are arranged in a square lattice of period $750 \mathrm{~nm}$ as shown in the inset. The dielectric film is uniformly magnetized perpendicularly to its plane. (b) Calculated transmittance (solid line) and 
Faraday rotation (dashed line) at wavelength $963 \mathrm{~nm}$ versus the thickness $h$ of the magnetic layer for the same magnetoplasmonic crystal as in (a). The dotted line represents the Faraday rotation for the same Bi:YIG layer placed in optically matched and non-structured surrounding medium. (c) Experimental cross-polarized transmission spectra at normal incidence for a magnetoplasmonic crystal (50-nm-thick Au film perforated with an array of holes of $125 \mathrm{~nm}$ diameter and $400 \mathrm{~nm}$ period deposited onto a $3.5 \mu \mathrm{m}$ thick single crystalline iron garnet film) in a varying external magnetic field $(0-80 \mathrm{mT})$ applied perpendicularly to the film. The polarization of the incident light is parallel to the $(1,0)$ axis of the crystal lattice. (a)-(b) are reproduced with permission from [166]. Copyright 2007, The American Physical Society. (c) is reproduced with permission from [169]. Copyright 2008, IOP Publishing Ltd. and Deutsche Physikalische Gesellschaft. 


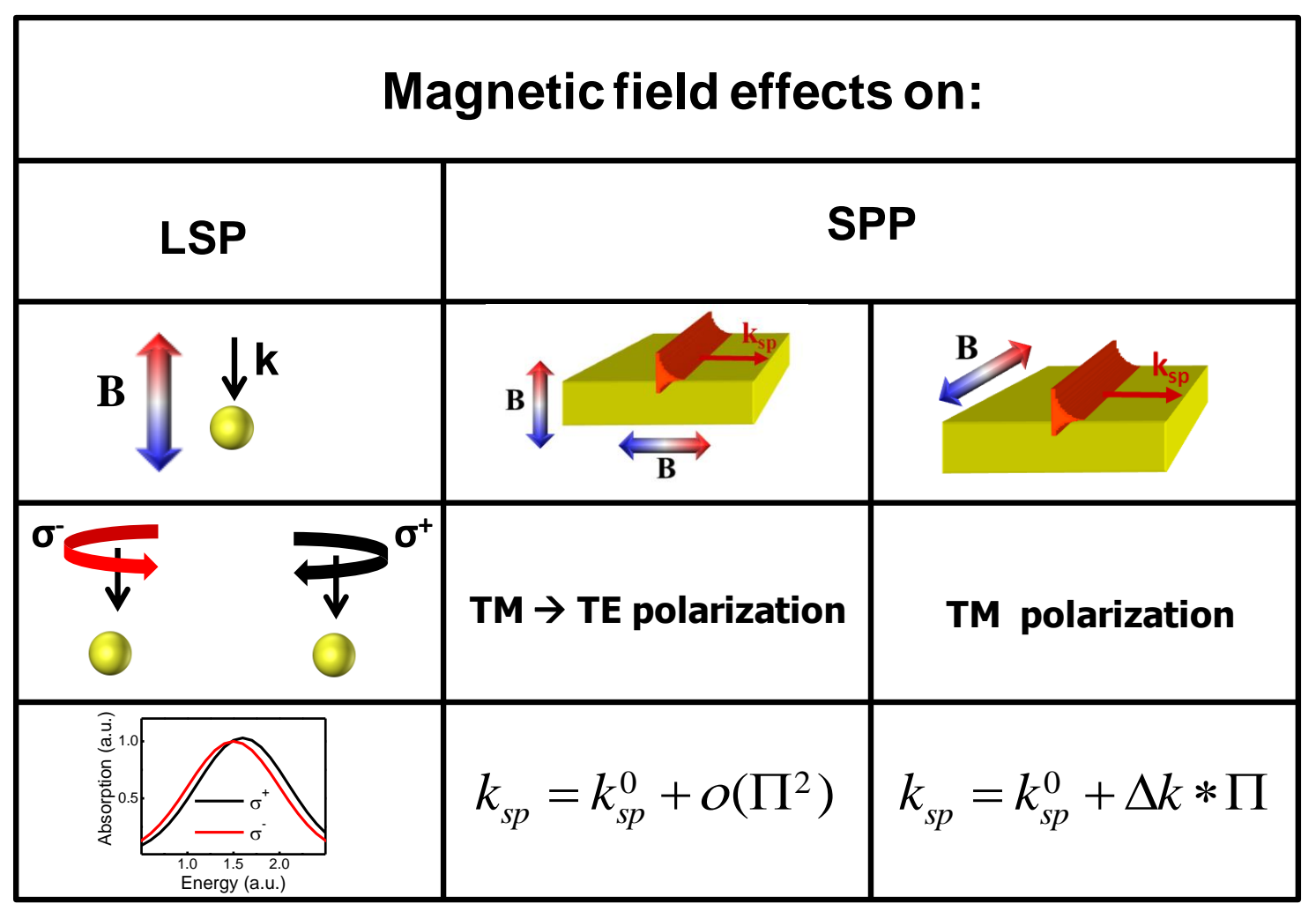

Figure 14. Left: Effect of an external applied magnetic field on a LSP resonance. For a spherical particle, when a magnetic field is applied parallel to the propagation direction of the light, the spectral position of the absorption peak is different for left-handed or right-handed circularly polarized light. Right: Sketch of the effect of an external magnetic field on SPPs. For polar and longitudinal configurations (middle) the effect on the SPP wavevector is very small and involves polarization conversion. For transversal configuration (bottom) there is no polarization conversion and the SPP wavevector varies linearly with $\Pi$. 

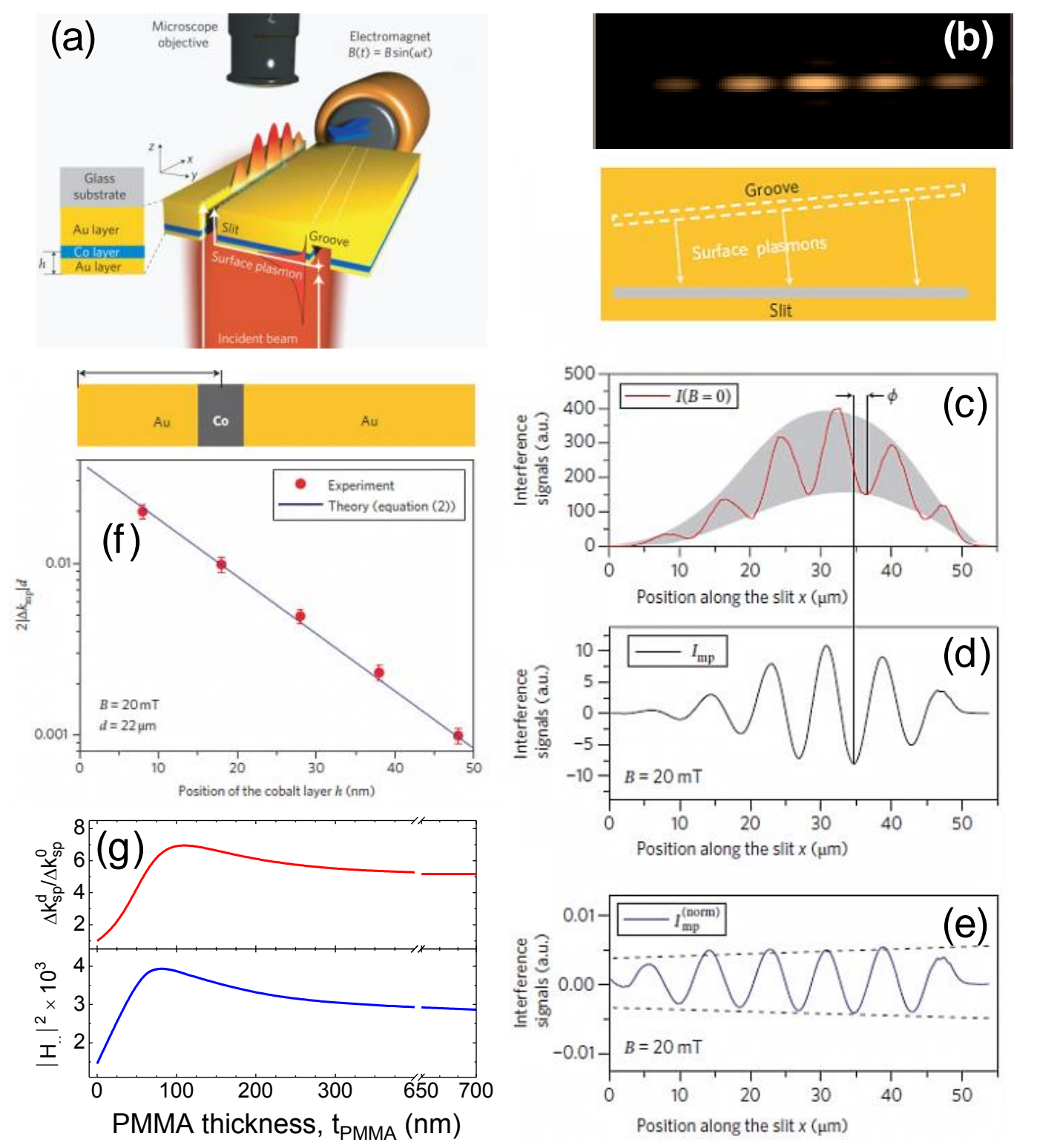

Figure 15. (a) Sketch of a magnetoplasmonic interferometer, consisting of a tilted slitgroove pair on a $\mathrm{Au} / \mathrm{Co} / \mathrm{Au}$ film. SPPs are generated at the groove by the incident light and propagate towards the slit, where they interfere with the light transmitted through the slit giving rise to a periodic interference pattern along the slit, as can be seen in the image shown in (b). An oscillating magnetic field is applied parallel to the interface and perpendicular to the SPP propagation direction which modifies the SPP wavevector and therefore modifies the interference pattern. (c) Optical interferogram, which corresponds to the DC component of the light intensity collected after passing through the slit. (d) Magnetoplasmonic interferogram, i.e. magnetic field induced changes of the 
collected light intensity. (e) Normalized magnetoplasmonic signal, obtained from (d) and (c). The magnetic field induced modulation of the SPP wavevector, $\Delta k_{s p}$, is obtained from slope of the dotted line. (f) Dependence of $\Delta k_{s p}$ on the position of the Co layer inside the multilayer, reflecting the exponential decay of the SPP electromagnetic field inside the metallic film. $\Delta k_{s p}$ can be increased by introducing a thin layer of dielectric on top of the interferometer, as can be seen in (g) for increasing PMMA thickness. The value of $\Delta k_{s p}$ closely follows the evolution of the electromagnetic field inside the cobalt layer. (a-f) are adapted with permission from [37]. Copyright 2010, Macmillan Publishers Ltd. (g) is adapted with permission from [193]. Copyright 2010, American Institute of Physics. 

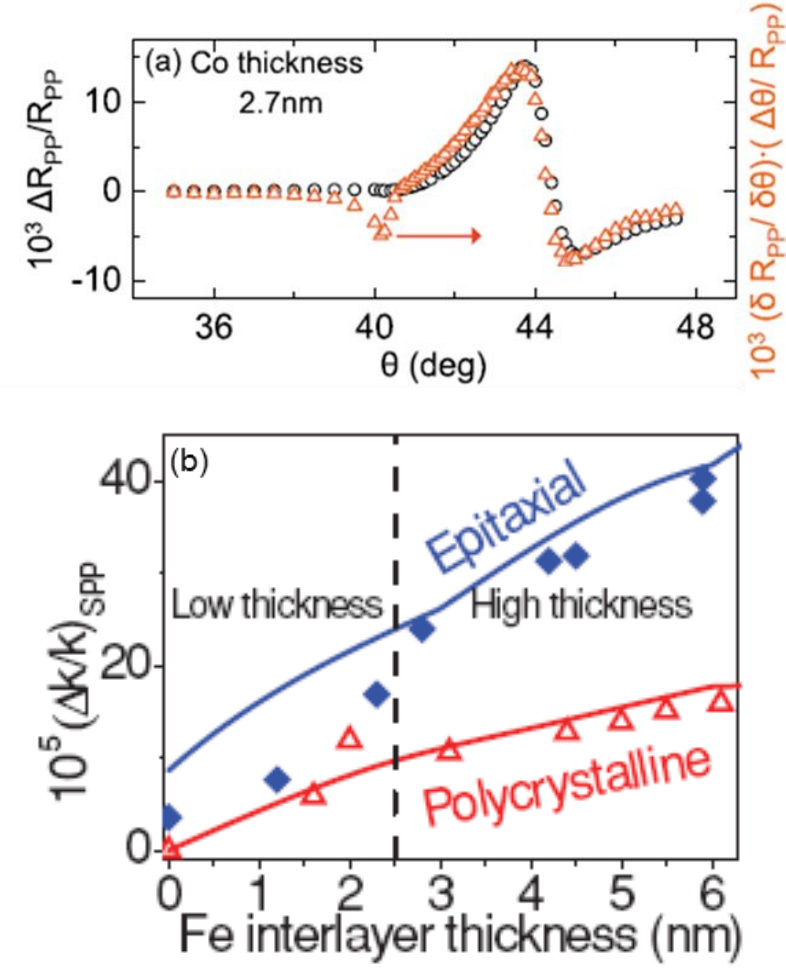

Figure 16. (a) Comparison between the transversal MO Kerr reflectivity (black dots) and the derivative of the reflectivity (red triangles) for a $6 \mathrm{~nm} \mathrm{Au} / 2.7 \mathrm{~nm} \mathrm{Co} / 16 \mathrm{~nm}$ $\mathrm{Au} /$ glass trilayer. The value of $\Delta \theta$ needed to match the peak-to-peak intensity of the derivative to that of the transversal MO Kerr signal allows to determine the wavevector modulation, $\Delta \mathrm{k}_{\mathrm{sp}}$. (b) Experimentally determined values of $\Delta \mathrm{k}_{\mathrm{sp}}$ for polycrystalline and epitaxial $7 \mathrm{~nm} \mathrm{Au} / d \mathrm{~nm} \mathrm{Fe} / 7 \mathrm{~nm} \mathrm{Au} /$ buffer layer/MgO(001) multilayers as a function of the Fe layer thickness, $d$. The polycrystalline structures are obtained by depositing a buffer layer of $2 \mathrm{~nm} \mathrm{Cr}$ by $\mathrm{MBE}$, whereas the epitaxial structures are obtained by depositing a buffer layer of $1 \mathrm{~nm}$ Fe by PLD. The better interface quality obtained in the epitaxial structures gives rise to a much stronger SPP wavevector modulation. (a) is reproduced with permission from [132]. Copyright 2007, The American Physical Society; (b) is reproduced with permission from [137]. Copyright 2011, American Physical Society. 


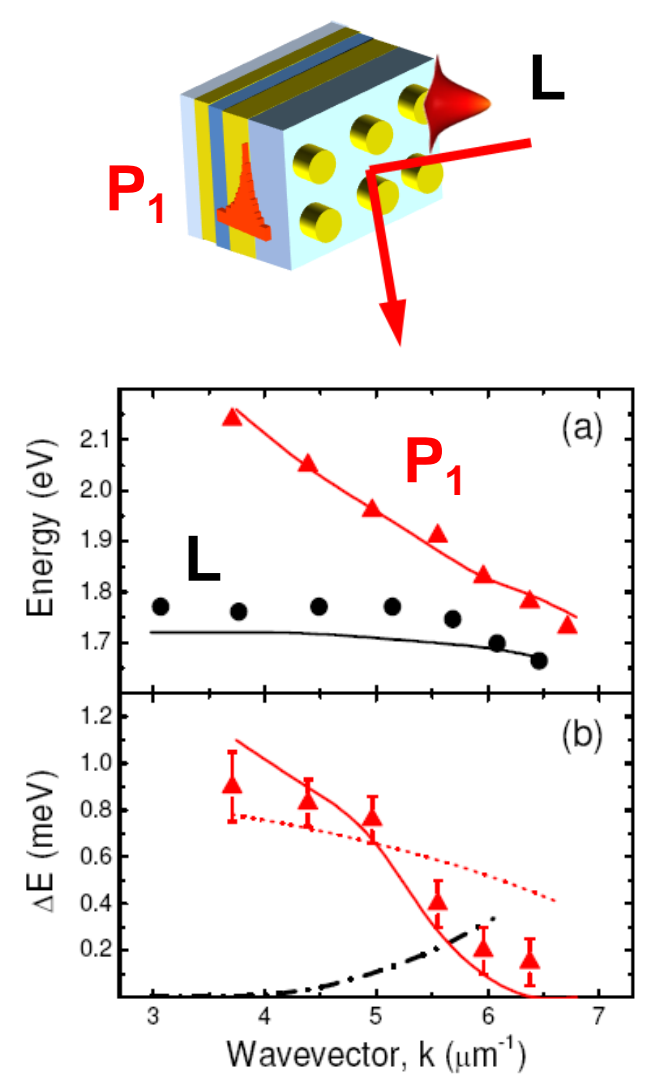

Figure 17. Sketch of the analyzed structure, a square array of gold nanodisks supporting LSPs (L) separated by a $\mathrm{SiO}_{2}$ spacer from a $\mathrm{Au} / \mathrm{Co} / \mathrm{Au}$ trilayer that supports SPPs. The excitation of the SPP is allowed by the periodicity of the disks array. This configuration permits the excitation of two SPPs, associated to each interface, being the most efficient that of the $\mathrm{Au} / \mathrm{SiO}_{2}$ spacer interface (P1). (a) Experimental (symbols) and theoretical (lines) dispersion relations of the LSP and SPP modes for the sample with an array of period $400 \mathrm{~nm}$. The interaction region is located around $6 \mu \mathrm{m}^{-1}$, where strong coupling takes place (anti-crossing). (b) The strong coupling modifies the magnetic-field effect on the plasmon modes wavevector $(\Delta \mathrm{E} \propto \Delta \mathrm{k})$ : LSP (dot-dashed black line, theory), SPP (red continuous line for theory, triangles for experimental data). The dotted red line shows, for comparison, the calculated modulation induced in the SPP sustained by a $\mathrm{SiO}_{2} / \mathrm{Au} / \mathrm{Co} / \mathrm{Au}$ multilayer system without disks array on top. Reproduced with permission from [197]. Copyright 2010, Optical Society of America. 

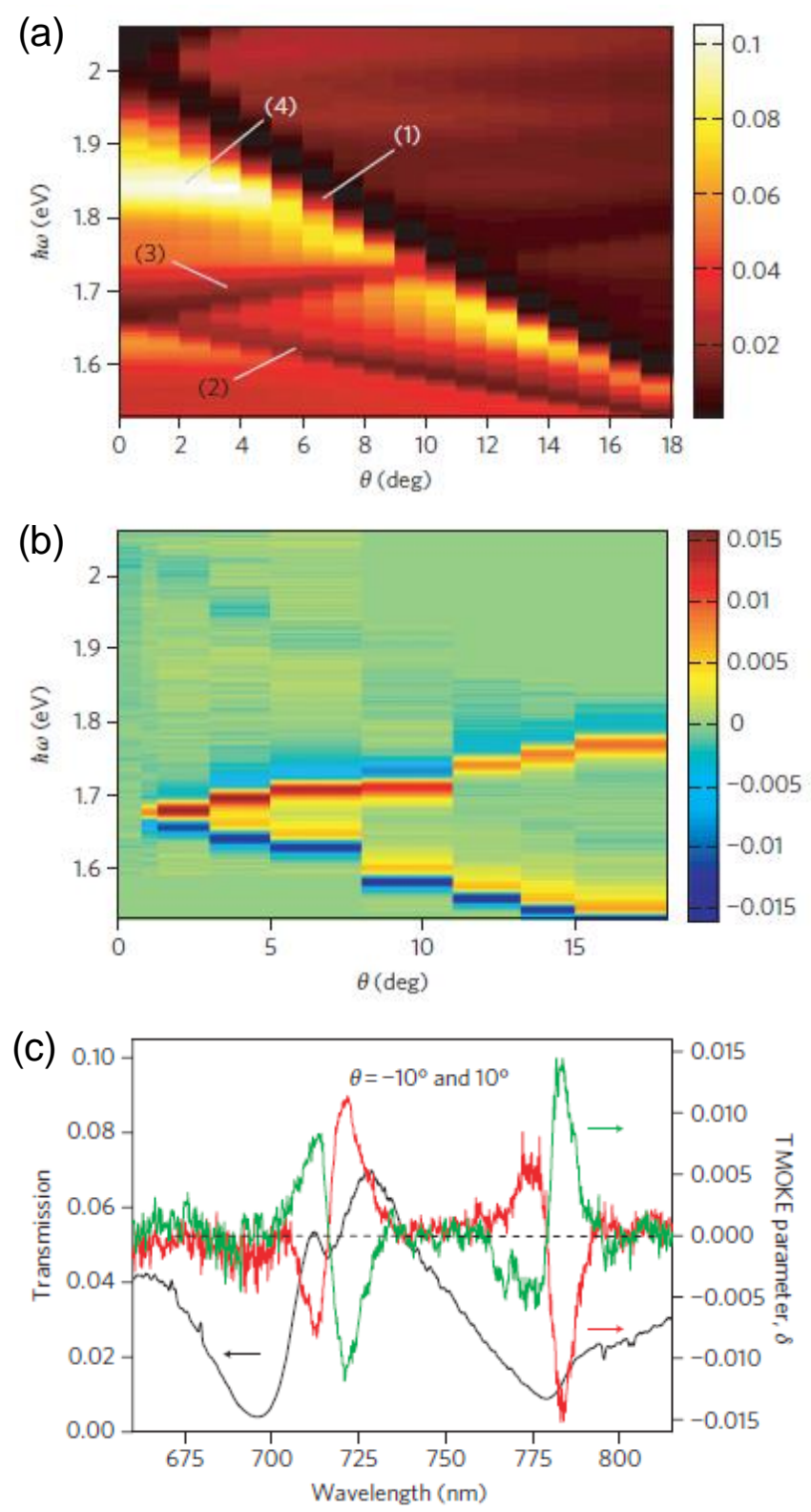

Figure 18. (a) Measured transmission as a function of the photon energy and the angle of incidence for a Au grating (grating period $=595 \mathrm{~nm}$, Au film height $=120 \mathrm{~nm}$ and slits width $=110 \mathrm{~nm}$ ) on top of a planar bismuth iron garnet film. The incidence light is p-polarized. Features marked as (1)-(4) correspond to: (1) excitation of the second-band SPP at the air/Au interface; (2)-(3) excitation of the second and third-band SPP at the Au/iron garnet interface; (4) collective Fabry-Perot cavity mode inside the slits. (b) Transversal MO Kerr reflectivity, $\Delta R_{p p} / R$, as a function of the photon energy and angle of incidence. The garnet film magnetization is parallel to the slits and the applied 
magnetic field strength is $0.2 \mathrm{~T}$, enough to saturate the film. (c) Transmission (black line) versus wavelength at angle of incidence $\theta=-10^{\circ}$ and transversal MO Kerr reflectivity at $\theta=-10^{\circ}$ (green line) and $\theta=10^{\circ}$ (red line). Reproduced with permission from [168]. Copyright 2011, Macmillan Publishers Ltd. 

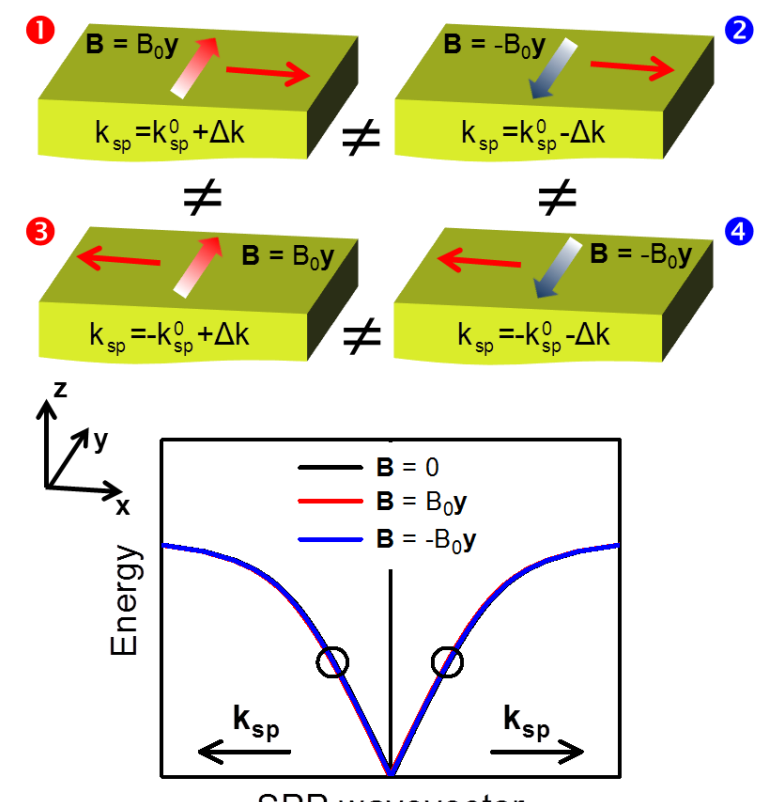

SPP wavevector
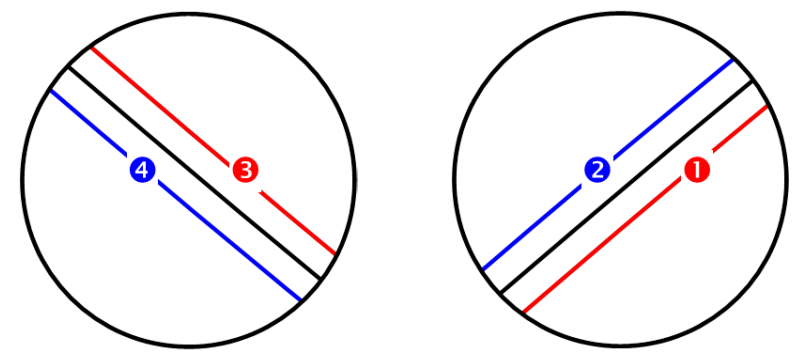

Figure 19. Sketch of SPP non-reciprocity. Under the same applied magnetic field, the modulus of the wavevector of a plasmon polariton propagating along one directions is different from that of a SPP travelling in the opposite way under the same applied magnetic field (schemes 1 and 3). Similarly, for a given SPP propagating along one direction, the wavevector changes when the applied magnetic field is reversed (schemes 1 and 2). In terms of dispersion relation, the non-reciprocity implies that, under the same applied magnetic field, the dispersion relation for propagating and counterpropagating plasmons is different, $\mathrm{w}(\mathbf{k}) \neq \mathrm{w}(-\mathbf{k})$. 
(a)

Electromagnet
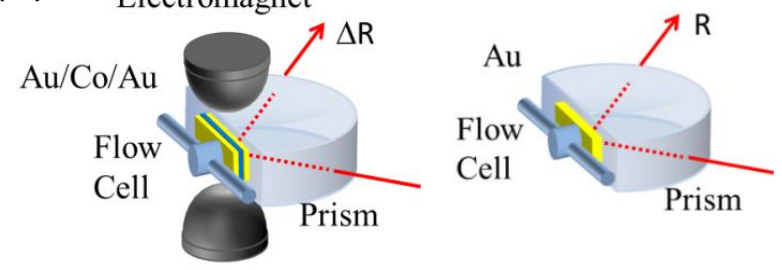

(b)
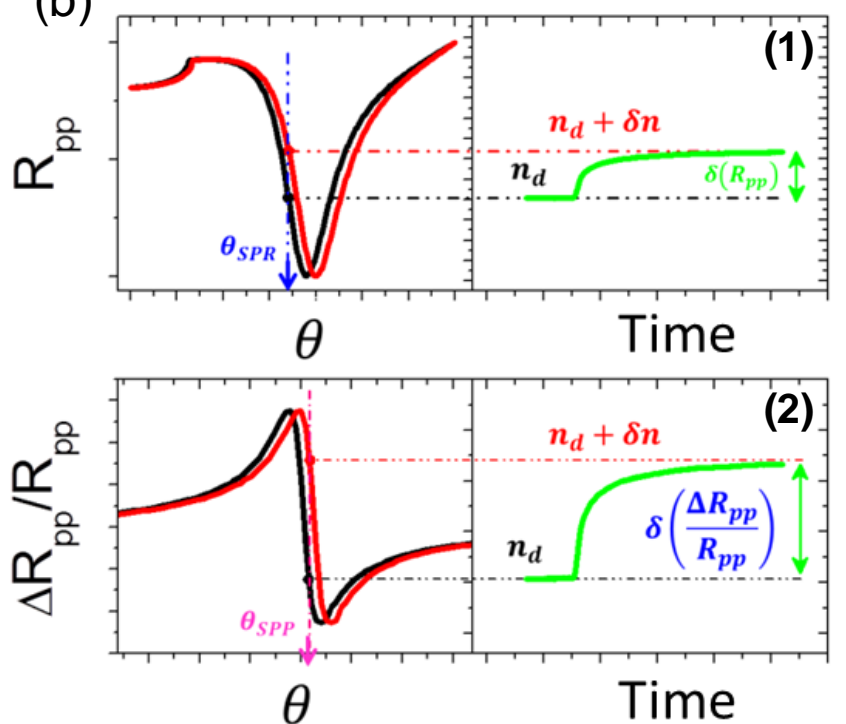

(c)

$\theta$

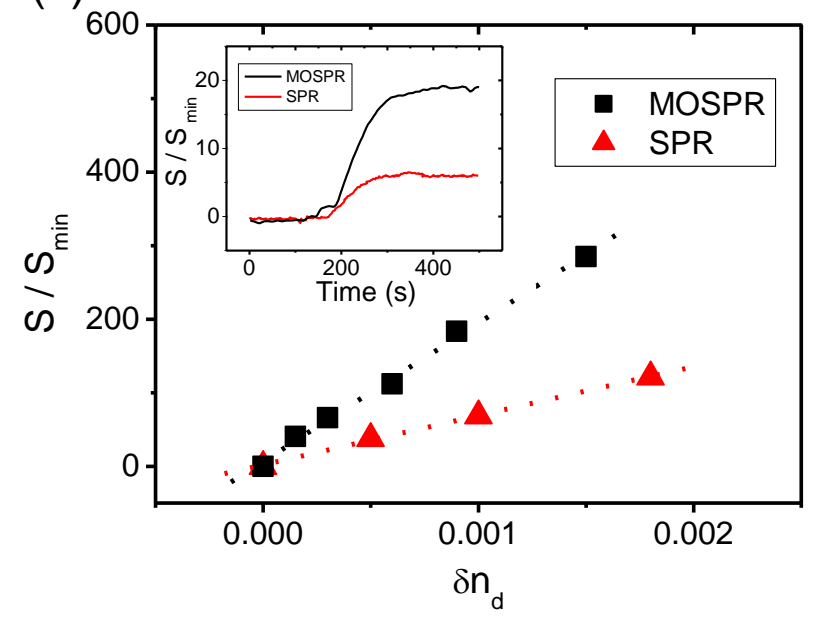

Figure 20. (a) Sketch of the MOSPR (left) and SPR (right) sensors. The experimental parts are very similar, the differences being the transducer, a Au layer for the SPR and a $\mathrm{Au} / \mathrm{Co} / \mathrm{Au}$ trilayer for the MOSPR, together with the application of an external magnetic field for the MOSPR. (b) Operating principle of the SPR (1) and MOSPR (2) biosensors: sensing response generated in a SPR sensor, $\delta R_{p p}$, or in a MOSPR one, $\delta\left(\Delta R_{p p} / R_{p p}\right)$, by a refractive index change of the dielectric medium, $\delta \mathrm{n}_{\mathrm{d}}$. (c) Comparison 
of the experimental normalized signals of the MOSPR and the SPR sensors due to refractive-index changes, and evaluation of their experimental sensitivities. The inset shows the normalized signal of the detection of the physical adsorption of bovine serum albumin proteins. (b) is adapted with permission from [74] and [208]. Copyright 2011, Optical Society of America. (c) is adapted with permission from [36]. Copyright 2006, Optical Society of America. 

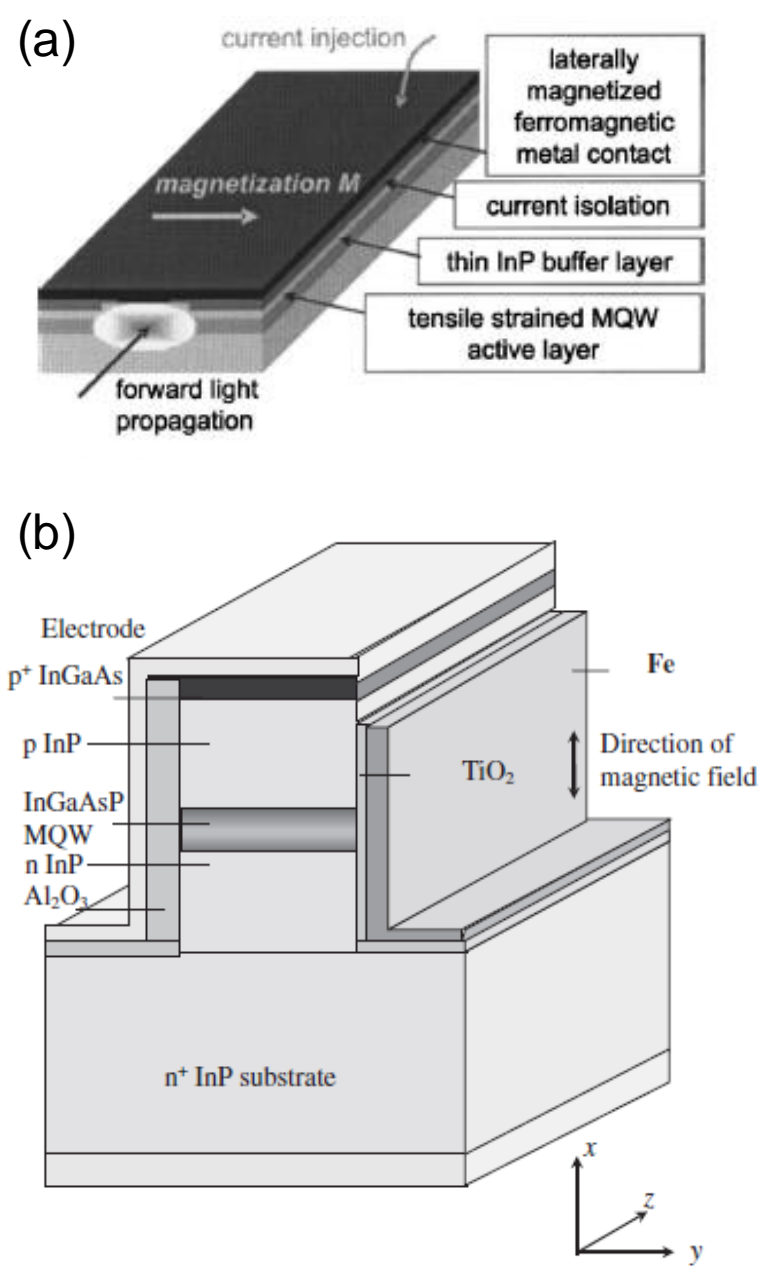

Figure 21. (a) Schematic structure of a TM mode optical waveguide isolator, with a ferromagnetic metal placed on top. (b) Schematic structure of the TE mode waveguide optical isolator, with a ferromagnetic metal layer placed on one sidewall. (a) is reproduced with permission from [214], Copyright 2006, American Institute of Physics; (b) is reproduced with permission from [215], Copyright 2004, The Japan Society of Applied Physics. 